\title{
THE TRANSFORMATION OF VECTOR-FUNCTIONS, SCALING AND BIFURCATION
}

\author{
BY \\ R. J. MAGNUS
}

\begin{abstract}
Various known methods for studying the bifurcation of zeros of a Banach-space-valued mapping are unified under a single idea, akin to using a coordinate transformation to obtain a simple form of the function under consideration. The general nature of the hypotheses permits the dropping of the pervasive "Fredholm condition" of bifurcation theory.
\end{abstract}

1. Introduction. Let $X$ and $Y$ be real Banach spaces and $g: X \rightarrow Y$ a $C^{n}$ mapping such that $g(0)=0$. We shall consider the problem of determining, up to diffeomorphism, the set of solutions of $g(x)=0$ in a neighbourhood of 0 . This problem arises in bifurcation theory, where solutions of an equation are sought near to a known degenerate solution.

To attack this question there exists a family of techniques scattered over hundreds of research papers. At the beginning is the implicit function theorem $(D g(0)$ surjective with complemented kernel), and various stages of degeneracy of $D g(0)$ have been studied usually by reducing the problem $g(x)=0$ to an algebraic problem in finitely many variables (the "bifurcation equations"). Later developments have received names like "the method of scaling" and "blowing up".

In this paper it is hoped to unify these methods under a single idea, that of transforming the function $g$ on an open set, of which 0 is in the closure, into a more amenable function $h$. More precisely we shall be concerned with a relationship between a pair of functions $g$ and $h$, which ensures the existence of open sets $E_{1}$ and $E_{2}$ and a diffeomorphism $\phi: E_{1} \rightarrow E_{2}$ such that $0 \in \bar{E}_{1} \cap \bar{E}_{2}$ and $g \phi=h \mid E_{1}$, the object being that the study of $g^{-1}(0)$ near to 0 is replaced by the study of $h^{-1}(0)$.

An earlier weaker version of the main theorem was proved by the author in an unpublished paper [7] which contained complicated proofs. This was intended to justify the result conjectured at the end of [6], proofs of which have since appeared in other articles $[\mathbf{1}, \mathbf{8}, \mathbf{9}, \mathbf{1 2}]$.

A great difficulty in bifurcation theory is the need for the Fredholm condition. This is the assumption that $D g(0)$ is a Fredholm operator. This condition is not needed in the main theorem. The dropping of the Fredholm condition in a concrete bifurcation problem has been the subject of a series of papers by Stuart; le.g. 13], but has been scarcely touched on elsewhere. In $\S 6$ we describe one way of extending the results of $[6]$ to a "non-Fredholm" case in that we permit $D g(0)$ to have nonclosed range, whereas the closure of the range is to have finite codimension.

Received by the editors April 11, 1983 and, in revised form, September 6, 1983.

1980 Mathematics Subject Classification. Primary 47H15, 58E07; Secondary 58C27.

(C) 1984 American Mathematical Society $0002-9947 / 84 \$ 1.00+\$ .25$ per page 
This work belongs to the no-man's-land between bifurcation theory and singularity theory, but is definitely inclined to the former. It is not resolved how it is related to the work of Kuiper and Kuo on $C^{0}$-equivalence $[\mathbf{4}, \mathbf{5}]$. This question will be touched on in the relevant places.

2. The main theorem. In this section we reprove Theorem 1.2 of $[\mathbf{7}]$. The theorem is slightly improved, but the proof is much shorter.

Let $X$ and $Y$ be real Banach spaces. We assume that $X$ and $Y$ are equipped with linear flows which we can write in the form $t \mapsto e^{t B}$ on $X$ and $t \mapsto e^{t C}$ on $Y$. The generators $B$ and $C$ are bounded linear operators on $X$ and $Y$ respectively, and we assume that the spectrum of the complexification of $B$ lies in the left half-plane and is bounded away from the imaginary axis. This implies that $\lim _{t \rightarrow \infty} e^{t B}=0$. We now set $\theta(\alpha)=e^{-(\log \alpha) B}$ and $\pi(\alpha)=e^{-(\log \alpha) C}$ where $\alpha>0$. From now on we forget about $B$ and $C$ and just use $\theta$ and $\pi$. Note that

(i) $\theta(\alpha \beta)=\theta(\alpha) \theta(\beta)=\theta(\beta) \theta(\alpha)$,

(ii) $\theta(1)=I$.

Similar properties are possessed by $\pi$. In addition $\lim _{\alpha \rightarrow 0+} \theta(\alpha)=0$.

The curve $\alpha \mapsto \theta(\alpha) x$ will be called the trajectory through $x$. The term $\theta$ invariant refers to the invariance of an object (set, vector-field etc.) under all transformations $\theta(\alpha)$ for $\alpha>0$.

Let $D$ be an open $\theta$-invariant subset of $X \backslash\{0\}$. A subset $E$ of $X \backslash\{0\}$ is called a $\theta$-set of $D$ if for every $x_{0} \in D$ there exist $r>0$ and a neighbourhood $U$ of $x_{0}$ such that $E$ contains all points $\theta(\alpha) x$ where $0<\alpha<r$ and $x \in U$.

If $f: X \backslash\{0\} \rightarrow Y$ we say that $f$ is $(\pi, \theta)$-homogeneous if for all $x \in X \backslash\{0\}$ and $\alpha>0$

$$
f(\theta(\alpha) x)=\pi(\alpha) f(x) .
$$

Now let $g$ and $h$ be $C^{n}$ mappings (for some fixed $n \geq 1$ ) from $X \backslash\{0\}$ into $Y$ and assume that

$$
\begin{gathered}
\lim _{\alpha \rightarrow 0+} \pi\left(\alpha^{-1}\right) g(\theta(\alpha) x)=h(x), \\
\lim _{\alpha \rightarrow 0+} \pi\left(\alpha^{-1}\right) D g(\theta(\alpha) x) \theta(\alpha)=D h(x),
\end{gathered}
$$

where the limits are locally uniform for $x \in X \backslash\{0\}$. Note that (2.2) is obtained by formally differentiating (2.1), and that $(2.1)$ implies that $h$ is $(\pi, \theta)$-homogeneous.

The prototype for the relations (2.1) and (2.2) is provided by the Morse Lemma. If $g: X \rightarrow \mathbf{R}$ is $C^{\infty}, g(0)=0, D g(0)=0$, we let $h(x)=\frac{1}{2} D^{2} g(0) x^{(2)}$. Then (2.1) and (2.2) are satisfied with $\theta(\alpha)=\alpha I$ and $\pi(\alpha)=\alpha^{2} I$. The Morse Lemma gives conditions under which $g=h \phi$ for a local diffeomorphism $\phi$ defined near 0 in $X$.

Although the Morse Lemma is a convenient motivation for the main result, there are profound differences, mainly because we do not assert that anything nondegenerate happens at $x=0$. Indeed $g$ need not be defined at 0 .

THEOREM 2.1. Let $g: X \backslash\{0\} \rightarrow Y$ and $h: X \backslash\{0\} \rightarrow Y$ be $C^{n}$ mappings which satisfy (2.1) and (2.2). Let $D$ be an open $\theta$-invariant subset of $X \backslash\{0\}$ such that

(i) $h \mid D$ is a submersion,

(ii) there exists a $C^{n}$ subbundle of the tangent bundle $T D$ which is $\theta$-invariant and is transversal to ker Dh. 
Then there exists a $C^{n}$ diffeomorphism $\phi: E \rightarrow \phi(E)$, where $E$ and $\phi(E)$ are $\theta$-sets of $D$, such that for all $x \in E, g(x)=h(\phi(x))$.

Furthermore on the trajectory through $x$ the diffeomorphism $\phi$ has the form

$$
\theta(\alpha) x \mapsto \theta(\alpha)(x+\eta(\alpha, x))
$$

where $\lim _{\alpha \rightarrow 0+} \eta(\alpha, x)=0$ locally uniformly with respect to $x$.

We preface the proof with some remarks.

(2.3) Condition (i) means that for each $x \in D$ the derivative $D h(x)$ is surjective and its kernel admits a topological complement.

(2.4) In the original version of this theorem [7] condition (ii) was replaced by a stronger requirement, equivalent to making the transversal subbundle an integrable distribution.

(2.5) If $X$ is finite dimensional and $h$ of class $C^{n+1}$ then (i) implies (ii). This is proved in Appendix 1.

PROOF. Let $F$ be the $\theta$-invariant subbundle of $T D$ transversal to ker $D h$. If $\alpha \in[0, \infty), x \in D$ and $u \in F_{x}$ we define

$$
\begin{aligned}
& M(\alpha, x, u)=\pi\left(\alpha^{-1}\right) g(\theta(\alpha) x)-h(x+u) \quad \text { if } \alpha>0, \\
& M(0, x, u)=h(x)-h(x+u) .
\end{aligned}
$$

Then $M$ is continuous on $[0, \infty) \times F, C^{n}$ on $(0, \infty) \times F$ and the partial derivatives $D_{2} M$ and $D_{3} M$ are continuous on $[0, \infty) \times F$. We have in fact

$$
D_{3} M(0, x, 0)=-D h(x) \mid F_{x}
$$

and this is an isomorphism of $F_{x}$ onto $Y$. For all $x \in D, M(0, x, 0)=0$. By the implicit function theorem there is an open set $B$ in $[0, \infty) \times D$ containing $\{0\} \times D$ and a mapping $\hat{u}: B \rightarrow F$ such that $\hat{u}(\alpha, x) \in F_{x}, \hat{u}$ is $C^{n}$ for $\alpha>0, D_{2} \hat{u}$ is continuous for $\alpha \geq 0$ and $M(\alpha, x, \hat{u}(\alpha, x))=0$ for all $(\alpha, x) \in B$. Moreover $\hat{u}(0, x)=0$ for all $x \in D$ and we can arrange that the domain $B$ should have the property that $(\alpha, x) \in B$ implies $(\alpha \beta, x) \in B$ for $0 \leq \beta \leq 1$. We define

$$
B_{+}=\{(\alpha, x) \in B: \alpha>0\} \text {. }
$$

Clearly we must define $\phi$ in such a way that

$$
\phi(\theta(\alpha) x)=\theta(\alpha)(x+\hat{u}(\alpha, x))
$$

for then

$$
g(\theta(\alpha) x)=\pi(\alpha) h(x+\hat{u}(\alpha, x))=h(\theta(\alpha)(x+\hat{u}(\alpha, x)))=h(\phi(\theta(\alpha) x)) .
$$

The problem is to show that $\theta(\alpha)(x+\hat{u}(\alpha, x))$ depends only on $\theta(\alpha) x$. Suppose then that $(\alpha, x)$ and $\left(\alpha_{1}, x_{1}\right)$ are points in $B_{+}$such that $\theta(\alpha) x=\theta\left(\alpha_{1}\right) x_{1}$. We have

$$
\pi\left(\alpha^{-1}\right) g(\theta(\alpha) x)-h(x+\hat{u}(\alpha, x))=0 .
$$

Operating with $\pi\left(\beta^{-1}\right)$ we find that

$$
\pi\left(\alpha^{-1} \beta^{-1}\right) g\left(\theta(\alpha \beta) \theta\left(\beta^{-1}\right) x\right)-h\left(\theta\left(\beta^{-1}\right) x+\theta\left(\beta^{-1}\right) \hat{u}(\alpha, x)\right)=0
$$

that is

$$
M\left(\alpha \beta, \theta\left(\beta^{-1}\right) x, \theta\left(\beta^{-1}\right) \hat{u}(\alpha, x)\right)=0
$$


Now $\theta\left(\beta^{-1}\right) \hat{u}(\alpha, x) \in F_{\theta\left(\beta^{-1}\right) x}$ by the $\theta$-invariance of $F$. Hence the uniqueness of the implicit function (see Appendix 2) gives

$$
\theta\left(\beta^{-1}\right) \hat{u}(\alpha, x)=\hat{u}\left(\alpha \beta, \theta\left(\beta^{-1}\right) x\right)
$$

whenever both sides are defined. Taking $\beta=\alpha_{1} \alpha^{-1}$ we find that

$$
\theta(\alpha) \hat{u}(\alpha, x)=\theta\left(\alpha_{1}\right) \hat{u}\left(\alpha_{1}, \theta\left(\alpha_{1}^{-1}\right) \theta(\alpha) x\right)=\theta\left(\alpha_{1}\right) \hat{u}\left(\alpha_{1}, x_{1}\right) .
$$

Thus $\phi$ is well defined and $C^{n}$ on the set $E_{0}=\left\{\theta(\alpha) x:(\alpha, x) \in B_{+}\right\}$. Obviously $E_{0}$ is a $\theta$-set of $D$. Moreover the continuity of $D_{2} \hat{u}$ for $\alpha \geq 0$ implies that $\lim _{\alpha \rightarrow 0+} \hat{u}(\alpha, x)=0$ locally uniformly with respect to $x$, and this gives us the last statement of the theorem.

Next we must construct the inverse of $\phi$. Using the property $\hat{u}(0, x)=0$ and the continuity of $D_{2} \hat{u}$ we can apply the implicit function theorem to the mapping $x \mapsto x+\hat{u}(\alpha, x)$. We obtain a unique function $f: C \rightarrow X$ where $C$ is an open subset of $[0, \infty) \times D$ containing $\{0\} \times D$, such that for all $(\alpha, x) \in C,(\alpha, f(\alpha, x)) \in B$ and

$$
f(\alpha, x)+\hat{u}(\alpha, f(\alpha, x))=x .
$$

In addition $C$ should have the property that if $(\alpha, x) \in C$ then $(\alpha \beta, x) \in C$ for $0 \leq \beta \leq 1$. We define

$$
C_{+}=\{(\alpha, x) \in C: \alpha>0\} \quad \text { and } \quad E_{1}=\left\{\theta(\alpha) x:(\alpha, x) \in C_{+}\right\} .
$$

Operating on $(* *)$ with $\theta\left(\beta^{-1}\right)$, and using $(*)$ and the uniqueness of $f$ we find that $\theta\left(\beta^{-1}\right) f(\alpha, x)=f\left(\alpha \beta, \theta\left(\beta^{-1}\right) x\right)$ whenever both sides are defined. Now let $\theta(\alpha) x=\theta\left(\alpha_{1}\right) x_{1}$ where both $(\alpha, x)$ and $\left(\alpha_{1}, x_{1}\right)$ are in $C_{+}$. We have that

$$
\theta(\alpha) f(\alpha, x)=\theta\left(\alpha_{1}\right) f\left(\alpha_{1}, \theta\left(\alpha_{1}^{-1} \alpha\right) x\right)=\theta\left(\alpha_{1}\right) f\left(\alpha_{1}, x_{1}\right) .
$$

Hence we may define $\psi$ on $E_{1}$ by $\psi(\theta(\alpha) x)=\theta(\alpha) f(\alpha, x)$ for all $(\alpha, x) \in C_{+}$. Let

$$
B_{+}^{\prime}=\left\{(\alpha, f(\alpha, x)):(\alpha, x) \in C_{+}\right\}
$$

If $(\alpha, x) \in B_{+}^{\prime}$ then $f(\alpha, x+\hat{u}(\alpha, x))=x$. We define the set $E$ of the theorem by $E=\left\{\theta(\alpha) x:(\alpha, x) \in B_{+}^{\prime}\right\}$. We finally check that $\phi \mid E$ and $\psi$ are inverses to each other. If $x \in E$ and $x=\theta(\alpha) y$ with $(\alpha, y) \in B_{+}^{\prime}$ then

$$
\psi(\phi(x))=\psi(\theta(\alpha)(y+\hat{u}(\alpha, y)))=\theta(\alpha) f(\alpha, y+\hat{u}(\alpha, y))=\theta(\alpha) y=x .
$$

If $x \in E_{1}$ and $x=\theta(\alpha) y$ with $(\alpha, y) \in C_{+}$then

$$
\phi(\psi(x))=\phi(\theta(\alpha) f(\alpha, y))=\theta(\alpha)(f(\alpha, y)+\hat{u}(\alpha, f(\alpha, y)))=\theta(\alpha) y=x .
$$

This concludes the proof.

3. Remarks on the main theorem. The simplest instances of Theorem 2.1 are those where $\theta(\alpha)$ is something obvious. We shall describe some of these and make further comments in a series of numbered paragraphs. Unexplained notation is the same as in Theorem 2.1.

(3.1) Let $g: X \rightarrow Y$ be a $C^{n}$ mapping such that $D^{j} g(0)=0$ for $j=0, \ldots, k-1$, where $k \leq n$. Then $(2.1)$ and (2.2) are satisfied with

$$
h(x)=\frac{1}{k !} D^{k} g(0) x^{(k)}, \quad \theta(\alpha)=\alpha I, \quad \pi(\alpha)=\alpha^{k} I .
$$


(3.2) If $K$ is a compact subset of $D$ then there exist $r>0$ and a neighbourhood $U$ of $K$ such that $E$ contains the set

$$
K_{0}=\{\theta(\alpha) x: 0<\alpha<r, x \in U\}
$$

and moreover the extension of $\phi$ to $K_{0} \cup\{0\}$ obtained by defining $\phi(0)=0$ is a homeomorphism. In particular if $D=X \backslash\{0\}$ and $X$ is finite dimensional then $E \cup\{0\}$ is a neighbourhood of 0 .

(3.3) If $X$ is finite dimensional, $D^{j} g(0)=0$ for $j=0, \ldots, k-1$, and the mapping $Q$ given by $Q(x)=D^{k} g(0) x^{(k)}$ is regular at all points $x \neq 0$, then there are neighbourhoods $U$ and $V$ of 0 in $X$ and a diffeomorphism $\phi: U \backslash\{0\} \rightarrow V \backslash\{0\}$ such that $g(x)=Q(\phi(x))$ for all $x \in U \backslash\{0\}$. It can be shown that $\phi$ can be extended to $U$ so as to be $C^{1}$ and satisfy $D \phi(0)=I$ (see $\S 4$ ).

(3.4) In (3.3) we could assume instead that $Q$ is regular on its zero-set (except at 0 ) and take $D$ to be a narrow cone containing $Q^{-1}(0)$. It then follows that the zero-sets of $g$ and $Q$ in a neighbourhood of 0 are homeomorphic. This is the conjectured theorem of $[6]$.

(3.5) The last paragraph combined with the Liapunov-Schmidt reduction gives information about $g^{-1}(0)$ for infinite-dimensional $X$. It is possible, however, to bypass Liapunov-Schmidt in such cases, and use Theorem 2.1 directly in infinite dimensions. This procedure is aesthetically appealing and was adopted in [7].

Suppose that $g$ is a $C^{n}$ mapping from $X$ into $Y$ such that $g(0)=0$ and $D g(0)$ is a Fredholm operator of nonnegative index. Let $X=V \oplus Z$ where $V=\operatorname{ker} D g(0)$. Suppose further that $D^{j} g(0) v^{(j)}=0$ for all $v \in V$ and $j=1, \ldots, k-1$, where $k \leq n$. Then we define

$$
h(x)=D g(0) z+\frac{1}{k !} D^{k} g(0) v^{(k)}
$$

where $x=v+z, v \in V$ and $z \in Z$. Define $\theta(\alpha) x=\alpha v+\alpha^{k} z, \pi(\alpha)=\alpha^{k} I$. Then $g$ and $h$ satisfy $(2.1)$ and (2.2).

Let $\bar{Y}=Y / D g(0) X$ and let $P: Y \rightarrow \bar{Y}$ be the canonical mapping. Let $B(v)=$ $P D^{k} g(0) v^{(k)}$ for $v \in V$. Note that $B$ is a mapping from $V$ into $\bar{Y}$ both of which are finite dimensional. We now assume that $B$ is regular on its zero-set (except at 0 ). Then $h$ is regular on its zero-set (except at 0 ).

We shall apply Theorem 2.1 where $D$ will be the set of regular points of $h$. The construction of a $\theta$-invariant subbundle $F$ in $T D$ transversal to $\operatorname{ker} D h$ is based on the following considerations. Identify $X$ with $V \times Z$. Then $D h\left(v_{0}, z_{0}\right)$ is the mapping

$$
(v, z) \mapsto D g(0) z+\frac{1}{(k-1) !} D^{k} g(0) v_{0}^{(k-1)} v .
$$

Then $D h\left(v_{0}, z_{0}\right)$ is surjective if and only if $D B\left(v_{0}\right)$ is surjective. Hence $D=$ $D_{1} \times Z$ where $D_{1}$ is the set of regular points of $B$. We construct a bundle $F_{1}$ over $D_{1}$, constant along rays through 0 and transversal to $\operatorname{ker} D B$. This is most easily done by introducing orthogonality in $V$; see also Appendix 1 . The bundle $F$ is defined fibre-by-fibre by $F_{(v, z)}=\left(F_{1}\right)_{v} \times Z$. Now we use Theorem 2.1 and obtain $\theta$-sets $E$ and $\phi(E)$ of $D$ and a diffeomorphism $\phi: E \rightarrow \phi(E)$ such that $g(x)=h(\phi(x))$. We would like to conclude that the set germs of $g^{-1}(0)$ and $h^{-1}(0)$ at 0 are homeomorphic. Unfortunately some additional arguments are now needed. Firstly $h^{-1}(0) \backslash\{0\}$ is of the form $\{\theta(\alpha) x: x \in K, 0<\alpha<\infty\}$ where $K$ is a compact 
subset of $D$. Referring now to (3.2) we find that $E$ contains a set $K_{0}$. Finally all zeros of $g$ sufficiently near to 0 lie in $K_{0}$ by Lemma $6(\mathrm{c})$ of $[6]$. Actually the germs of $g^{-1}(0)$ and $B^{-1}(0)$ are homeomorphic, since those of $B^{-1}(0)$ and $h^{-1}(0)$ are obviously homeomorphic. See also remark (3.7).

(3.6) The method of scaling, as developed in [2] corresponds to the case when $X$ is a direct sum of $\theta$-invariant subspaces $X_{1}, \ldots, X_{p}$ and on $X_{i}$ the operator $\theta(\alpha)$ is multiplication by $\alpha^{k_{i}}$ for positive $k_{1}, \ldots, k_{p}$. See also $\S 7$.

(3.7) The splitting of the space $X$ in remark (3.5) can be cast into a more general form. We thereby obtain a kind of extension of the Liapunov-Schmidt procedure.

Let $g: X \rightarrow Y$ be a $C^{2}$ mapping. Suppose that $X=V \oplus Z$ and that $g$ satisfies on $V$ relations like $(2.1)$ and (2.2) with certain trajectories in $V$. More precisely we assume that there are operators $\theta_{0}(\alpha)$ for $\alpha>0$ on $V$ having the properties required for $\theta(\alpha)$ in $\S 2$, and a $C^{2}$ mapping $f: V \rightarrow Y$ such that

$$
\begin{gathered}
\lim _{\alpha \rightarrow 0+} \alpha^{-k} g\left(\theta_{0}(\alpha) v\right)=f(v), \\
\lim _{\alpha \rightarrow 0+} \alpha^{-k} D g\left(\theta_{0}(\alpha) v\right) \theta_{0}(\alpha)=D f(v)
\end{gathered}
$$

locally uniformly for $v \in V \backslash\{0\}$. Then we define on $X$

$$
\theta(\alpha) x=\theta_{0}(\alpha) v+\alpha^{k} z
$$

where $x=v+z, v \in V, z \in Z$. We set $h(x)=D g(0) z+f(v)$. Then relations (2.1) and (2.2) are satisfied with $\pi(\alpha)=\alpha^{k} I$.

To see this we first observe that $g(v+z)-g(v)=T(v, z) z$ where $v \in V, z \in Z$ and $T: V \times Z \rightarrow L(Z, Y)$ is a $C^{1}$ mapping such that $T(0,0)=D g(0) \mid Z$. We shall verify (2.2). For arbitrary $v$ and $\bar{v}$ in $V$, and $z$ and $\bar{z}$ in $Z$ we have

$$
D g(v+z)(\bar{v}+\bar{z})-D g(v) \bar{v}=(D T(v, z)(\bar{v}, \bar{z})) z+T(v, z) \bar{z} .
$$

Hence

$$
\begin{gathered}
\alpha^{-k} D g\left(\theta_{0}(\alpha) v+\alpha^{k} z\right)\left(\theta_{0}(\alpha) \bar{v}+\alpha^{k} \bar{z}\right)-\alpha^{-k} D g\left(\theta_{0}(\alpha) v\right) \theta_{0}(\alpha) \bar{v} \\
=\left(D T\left(\theta_{0}(\alpha) v, \alpha^{k} z\right)\left(\theta_{0}(\alpha) \bar{v}, \alpha^{k} \bar{z}\right)\right) z+T\left(\theta_{0}(\alpha) v, \alpha^{k} z\right) \bar{z}
\end{gathered}
$$

Taking the limit as $\alpha \rightarrow 0$ we obtain (2.2). The verification of (2.1) is even simpler (but see remark (3.8)).

We shall next prove that if $V$ is finite dimensional and $D g(0) \mid Z$ has a bounded inverse, then small solutions of $g(x)=0$ lie near to solutions of $h(x)=0$. The special case when $\theta_{0}(\alpha)=\alpha$ and $V=\operatorname{ker} D g(0)$ was spread over several lemmata and theorems in [6]. The present result is more general and the proof much more concise. We shall need a hyperellipsoid $S$ in $V$ enclosing 0 with the property that each trajectory meets it in a unique point. The existence of $S$ is shown in Appendix 1.

THEOREM 3.1. Let $V$ be finite dimensional and $D g(0) \mid Z$ have a bounded inverse. Then $\lim _{x \rightarrow 0}\|D g(0) z+f(u)\|=0$ where the limit is taken for $x \in g^{-1}(0)$ and $x=\theta_{0}(\alpha) u+\alpha^{k} z$ for $u \in S$ and $z \in Z$.

ProOF. Let $K$ be such that $K\|z\| \leq\|D g(0) z\|$ for all $z \in Z$. Choose a neighbourhood $N$ of 0 in $X$ such that if $x \in N$ then

$$
\left\|\alpha^{-k} g\left(\theta_{0}(\alpha) u\right)-f(u)\right\| \leq \frac{1}{2} K \quad \text { and } \quad\left\|T\left(\theta_{0}(\alpha) u, \alpha^{k} z\right)-D g(0)\right\| \leq \frac{1}{2} K .
$$


Recall that $g(v+z)-g(v)=T(v, z) z$ and so

$$
\alpha^{-k} g\left(\theta_{0}(\alpha) u+\alpha^{k} z\right)-\alpha^{-k} g\left(\theta_{0}(\alpha) u\right)=T\left(\theta_{0}(\alpha) u, \alpha^{k} z\right) z
$$

Hence if $x \in N \cap g^{-1}(0)$ we have

$$
\|D g(0) z+f(u)\| \leq \frac{1}{2} K+\frac{1}{2} K\|z\|
$$

and so $\frac{1}{2} K\|z\| \leq \frac{1}{2} K+\|f(u)\|$. So $\|z\|$ is bounded by a constant $C$ if $x \in N \cap g^{-1}(0)$. Now given $\varepsilon>0$ choose a neighbourhood $N_{0} \subset N$ such that if $x \in N_{0}$ we have

$$
\left\|\alpha^{-k} g\left(\theta_{0}(\alpha) u\right)-f(u)\right\| \leq \frac{1}{2} \varepsilon \quad \text { and } \quad\left\|T\left(\theta_{0}(\alpha) u, \alpha^{k} z\right)-D g(0)\right\| \leq \frac{1}{2} \varepsilon / C .
$$

Then for $x \in N_{0} \cap g^{-1}(0)$ we find that

$$
\|D g(0) z+f(u)\| \leq \frac{1}{2} \varepsilon+\frac{1}{2} \varepsilon\|z\| / C \leq \varepsilon .
$$

This concludes the proof.

Now let $P: Y \rightarrow Y / D g(0) Z$ be the natural mapping and let $B=P \circ f \mid V$. Let us assume that all zeros of $B$, excepting 0 , are regular. Then under the hypotheses of Theorem 3.1, we have the result that the set germs of $g^{-1}(0)$ and $B^{-1}(0)$ are homeomorphic, just as in remark (3.5).

(3.8) In many cases the limit formulae (2.1) and (2.2) are not independent, so that it is only necessary to verify one of them.

If $g$ and $h$ are continuous on neighbourhoods of 0 and $g(0)=h(0)=0$, then, provided (2.2) holds we have that

$$
\begin{aligned}
\pi(\alpha)^{-1} g(\theta(\alpha) x)-h(x) \\
\quad=\int_{0}^{1}(\pi(t) / t)\left[\pi(\alpha t)^{-1} D g(\theta(\alpha t) x) \theta(\alpha t)-D h(x)\right](-B) x d t
\end{aligned}
$$

whence it follows that (2.2) implies (2.1) if, in addition $\|\pi(t)\| / t$ is integrable on $(0,1)$. This nearly always holds in practice, though it is unclear whether it may be dispensed with in general.

As (2.2) is more complicated than (2.1) it is preferable to have conditions under which (2.1) implies (2.2). The following conditions, taken together, are sufficient.

(i) $\pi\left(\alpha^{-1}\right)=\alpha^{-k} \pi_{1}(\alpha)$ where $\pi_{1}$ is $C^{k+1}$ on $[0, \infty)$.

(ii) $g$ is $C^{k+1}$ on a neighbourhood of 0 .

(iii) $\theta$ is $C^{k+1}$ on $[0, \infty)$.

We then have, if (2.1) holds,

$$
h(x)=\frac{1}{k !} D_{\alpha}^{k}\left[\pi_{1}(\alpha) g(\theta(\alpha) x)\right]_{\alpha=0}
$$

so that $h(x)$ is a polynomial. The implication in question then reduces to permuting mixed derivatives of order $k+1$.

(3.9) Let us assume that $g$ is $C^{k+m}$ on a neighbourhood of 0 , that $\theta$ is $C^{k+m}$ on $[0, \infty)$, and that $\pi\left(\alpha^{-1}\right)=\alpha^{-k} \pi_{1}(\alpha)$ where $\pi_{1}$ is $C^{k+m}$ on $[0, \infty)$. Then, referring to the proof of Theorem 2.1, we have

$$
M(\alpha, x, u)=\alpha^{-k} \pi_{1}(\alpha) g(\theta(\alpha) x)-h(x+u)
$$

so that $M$ is $C^{m}$ on $[0, \infty) \times F$. Hence $\hat{u}$ is $C^{m}$ for $\alpha \geq 0$. The extra differentiability of $\hat{u}$ will be useful later. 
4. Differentiability of $\phi$ at the origin. Throughout this section we shall consider $\phi$ as extended to $E \cup\{0\}$ by defining $\phi(0)=0$. One of our principal conclusions will be that $\phi$ is $C^{1}$ if $E \cup\{0\}$ is a neighbourhood of 0 and $g$ differs from $h$ by sufficiently small terms.

Assume that there exists $\sigma \geq 0$ such that

$$
\pi\left(\alpha^{-1}\right) g(\theta(\alpha) x)-h(x)=o\left(\alpha^{\sigma}\right)
$$

locally uniformly for $x \neq 0$, and

$$
\|\theta(\alpha)\| \cdot\left\|\theta\left(\alpha^{-1}\right)\right\|=O\left(\alpha^{-\sigma}\right)
$$

as $\alpha \rightarrow 0$. We observe that (4.2) will hold if $\theta(\alpha)$ corresponds to scaling (see remark (3.6)) with exponents $k_{1}, \ldots, k_{m}$ and $\sigma=\max \left|k_{i}-k_{j}\right|$. Equations (4.1) and (2.6) now imply $h(x+\hat{u}(\alpha, x))-h(x)=o\left(\alpha^{\sigma}\right)$ and so because $h$ is a submersion and $\hat{u}(\alpha, x) \in F_{x}$ we have $\hat{u}(\alpha, x)=o\left(\alpha^{\sigma}\right)$ locally uniformly for $x \neq 0$. Hence

$$
\|\phi(\theta(\alpha) x)-\theta(\alpha) x\| /\|\theta(\alpha) x\| \leq\|\theta(\alpha) \hat{u}(\alpha, x)\| \cdot\left\|\theta\left(\alpha^{-1}\right)\right\| /\|x\|
$$

so that we have

$$
\|\phi(\theta(\alpha) x)-\theta(\alpha) x\| /\|\theta(\alpha) x\| \rightarrow 0
$$

as $\alpha \rightarrow 0$, locally uniformly for $x \neq 0$.

In a certain sense therefore $D \phi(0)$ is the identity. In fact for any $x_{0} \neq 0$ in the domain of $\phi$, there is a neighbourhood $U$ of $x_{0}$ such that if $y$ tends to 0 inside the cone $\{\theta(\alpha) x: 0<\alpha \leq 1, x \in U\}$ then $\|\phi(y)-y\| /\|y\|$ tends to 0 . This means that $\phi$ preserves the tangent at 0 of any curve through 0 which lies in such a cone, though, be it noted, not all smooth curves through 0 need do so. Furthermore if $E \cup\{0\}$ contains a finite-dimensional submanifold $N$ containing 0 and invariant under $\theta(\alpha)$ for $0<\alpha \leq 1$, then the restriction of $\phi$ to $N$ has derivative the identity at 0 in the usual sense. In particular if $X$ is finite dimensional and if 0 is an interior point of $E \cup\{0\}$ then $D \phi(0)=I$, assuming of course (4.1) and (4.2).

We must also consider what technical assumptions in addition to (4.1) and (4.2) ensure continuity of $D \phi(x)$ at $x=0$. We have that

$$
\phi(\theta(\alpha) x)=\theta(\alpha)(x+\hat{u}(\alpha, x))
$$

and hence

$$
D \phi(\theta(\alpha) x)=I+\theta(\alpha) D_{2} \hat{u}(\alpha, x) \theta\left(\alpha^{-1}\right)
$$

so that

$$
\|D \phi(\theta(\alpha) x)-I\| \leq\|\theta(\alpha)\| \cdot\left\|D_{2} \hat{u}(\alpha, x)\right\| \cdot\left\|\theta\left(\alpha^{-1}\right)\right\| .
$$

To ensure that $D \phi(\theta(\alpha) x) \rightarrow I$ as $\alpha \rightarrow 0$ (locally uniformly for $x \neq 0$ ) it is enough if $D_{2} \hat{u}(\alpha, x)=o\left(\alpha^{\sigma}\right)$ (locally uniformly for $x \neq 0$ ) and this will follow if

$$
\pi\left(\alpha^{-1}\right) D g(\theta(\alpha) x) \theta(\alpha)-D h(x)=o\left(\alpha^{\sigma}\right)
$$

locally uniformly for $x \neq 0$, and in case $\sigma \neq 0$ we require $h$ to be of class $C^{2}$. This may be seen as follows. Since

$$
\pi\left(\alpha^{-1}\right) g(\theta(\alpha) x)=h(x+\hat{u}(\alpha, x))
$$

we have

$$
\pi\left(\alpha^{-1}\right) D g(\theta(\alpha) x) \theta(\alpha)=D h(x+\hat{u}(\alpha, x))\left(I+D_{2} \hat{u}(\alpha, x)\right)
$$


so that by $(4.3)$

$$
D h(x+\hat{u}(\alpha, x)) D_{2} \hat{u}(\alpha, x)=D h(x)-D h(x+\hat{u}(\alpha, x))+o\left(\alpha^{\sigma}\right) .
$$

We want the right-hand side to be $o\left(\alpha^{\sigma}\right)$. We already know that $\hat{u}(\alpha, x)$ is $o\left(\alpha^{\sigma}\right)$. To conclude that $D h(x)-D h(x+\hat{u}(\alpha, x))$ is $o\left(\alpha^{\sigma}\right)$ requires something more than continuity of $D h$ when $\sigma>0$, although to require $h$ to be $C^{2}$ is excessive.

Under the conditions (4.1), (4.2) and (4.3), and with $h$ of class $C^{2}$ if $\sigma>0$, we can conclude that on any finite-dimensional submanifold of $E \cup\{0\}$ containing 0 and invariant under $\theta(\alpha)$ for $0<\alpha \leq 1$, the restriction of $\phi$ is $C^{1}$.

In many cases (4.1) and (4.3) are not independent. If the mapping $(\alpha, x) \mapsto$ $\pi\left(\alpha^{-1}\right) g(\theta(\alpha) x)$ is $C^{m}$ for $\alpha \geq 0$ and $m \geq \sigma+1$ then (4.1) implies (4.3). Frequently $m=\infty$.

In case $\theta(\alpha)=\alpha I$ then $\sigma=0$ and (4.1), (4.2) and (4.3) are certainly fulfilled.

EXAMPLE. Let $h: \mathbf{R}^{n} \rightarrow \mathbf{R}$ be a polynomial such that $h(0)=0, D h(0)=0$ and $D h(x) \neq 0$ for $x \neq 0$. We suppose that $h(\theta(\alpha) x)=\alpha^{k} h(x)$ where $\theta(\alpha)\left(x_{1}, \ldots, x_{n}\right)=$ $\left(\alpha^{k_{1}} x_{1}, \ldots, \alpha^{k_{n}} x_{n}\right)$ for positive powers $k, k_{1}, k_{2}, \ldots, k_{n}$. Let $\sigma=\max \left|k_{i}-k_{j}\right|$. Now let $g(x)=h(x)+x^{t}$ where $x^{t}=x_{1}^{t_{1}} \cdots x_{n}^{t_{n}}, t=\left(t_{1}, \ldots, t_{n}\right)$ for positive $t_{1}, \ldots, t_{n}$. According to Theorem 2.1 we have $g(x)=h(\phi(x))$ for a homeomorphism $\phi$ in a neighbourhood of 0 whenever $k_{1} t_{1}+\cdots+k_{n} t_{n}>k$, and $\phi$ is a $C^{1}$ diffeomorphism with $D \phi(0)=I$ whenever $k_{1} t_{1}+\cdots+k_{n} t_{n}>k+\sigma$ according to the present section. Moreover if $r=\min \left\{k_{1}, \ldots, k_{n}\right\}$ then $h$ is $[k / r]$-determinate for $C^{0}$-equivalence, and $[(k+\sigma) / r]$-determinate for $C^{1}$-equivalence. The square bracket indicates integer part, and we suppose all functions to be $C^{\infty}$.

It is interesting to compare this with the results of Kuiper on $C^{0}$ and $C^{1}$ equivalence [4, p. 202, Theorems 1 and 2]. It is not hard to see that under the conditions of this example $\|D h(x)\| \geq c\|x\|^{(k / r)-1}$ whence the results of Kuiper and Kuo imply that $h$ is $[k / r]$-determinate for $C^{0}$-equivalence, so that we do not improve their conclusion. But for $C^{1}$-equivalence we can often do better. For example if $h(x)=x_{1}^{2}+x_{2}^{3}+\cdots+x_{n}^{n+1}$ then according to Kuiper $h$ is $2 n$-determinate and according to us it is $[(3 n+1) / 2]$-determinate for $C^{1}$-equivalence. Here we take $k=n+1$ and $k_{i}=(n+1) /(i+1)$. Of course Kuiper's results apply to a wider class of functions, on the face of it at least.

5. Extension of the main theorem: Uniformity. Even if $D \cup\{0\}$ is a neighbourhood of 0 it need not be the case that $E \cup\{0\}$ is a neighbourhood of 0 if $X$ is infinite dimensional. We can, however, achieve this by introducing more uniformity into the hypotheses of Theorem 2.1, and using a stronger form of the implicit function theorem (see Appendix 2).

We now make the following assumption which we call condition $(\mathrm{P})$, for "polar".

There exists a subset $S$ of $D$ such that the collection of sets $\left\{N_{r}: r>0\right\}$ defined by

$$
N_{r}=\{0\} \cup\{\theta(\alpha) w: 0<\alpha<r, w \in S\}
$$

forms a base for the system of neighbourhoods of 0 in $D \cup\{0\}$. 
In finite dimensions conditions (P) is always fulfilled, and $S$ may be chosen to be a hyperellipsoid enclosing the origin (see Appendix 1).

It is our aim to find conditions which ensure that $E \cup\{0\}$ is a neighbourhood of 0 in $D \cup\{0\}$. This will require a reexamination of the proof of Theorem 2.1. Essentially what is needed is to make the hypotheses of Theorem 2.1 hold uniformly for $x \in S$.

THEOREM 5.1. In addition to the hypotheses of Theorem 2.1 we make the following assumptions.

(i) Assumption (P).

(ii) $\left\|\left(D h(x) \mid F_{x}\right)^{-1}\right\|$ is bounded for $x \in S$.

(iii) $D h$ is uniformly continuous for $x \in S$.

(iv) Given $\varepsilon>0$ there exists $\delta>0$ such that

$$
\left\|\pi\left(\alpha^{-1}\right) g(\theta(\alpha) x)-h(x)\right\|<\varepsilon
$$

whenever $0<\alpha<\delta$ and $x \in S_{\delta}$, where $S_{\delta}$ is the set of points within $\delta$ of $S$.

(v) Given $\varepsilon>0$ there exists $\delta>0$ such that

$$
\left\|\pi\left(\alpha^{-1}\right) D g(\theta(\alpha) x) \theta(\alpha)-D h(x)\right\|<\varepsilon
$$

whenever $0<\alpha<\delta$ and $x \in S_{\delta}$.

Then the set $E$ given by Theorem 2.1 may be chosen so that both $E \cup\{0\}$ and $\phi(E) \cup\{0\}$ are neighbourhoods of 0 in $D \cup\{0\}$.

PROOF. We use the notation of the proof of Theorem 2.1. Recall that

$$
M(\alpha, x, u)=\pi\left(\alpha^{-1}\right) g(\theta(\alpha) x)-h(x+u), \quad M(0, x, u)=h(x)-h(x+u),
$$

where $\alpha \in[0, \infty), x \in D$ and $u \in F_{x}$. Now we use the uniform implicit function theorem (see $\S 10)$ at each point $(0, x, 0)$ with $x \in S$. We find that the domain of $\hat{u}$ which we called $B$ can be chosen to contain a set of the form $[0, r) \times S_{r}$. Furthermore the continuity of $\hat{u}$ and $D_{2} \hat{u}$ at points of the form $(0, x)$ is uniform for $x \in S$. This follows from the last two sentences of Theorem 10.2, and incidentally, condition (v) is needed only to obtain the conclusion about $D_{2} \hat{u}$. Now we apply the second part of Theorem 10.3 to the mapping $(\alpha, x) \mapsto x+\hat{u}(\alpha, x)$.

We find that there is a subset $C$ of the domain of $\hat{u}$ and $\delta>0$ such that the restriction of the mapping

$$
(\alpha, x) \mapsto(\alpha, x+\hat{u}(\alpha, x))
$$

to $C$ is a homeomorphism onto $[0, \delta) \times S_{\delta}$. Furthermore $C$ contains a set of the form $[0, \rho) \times S_{\rho}$. We now define $E$ as the set $E=\{\theta(\alpha) x:(\alpha, x) \in C\}$. Just as in the proof of Theorem 2.1 we may define $\phi$ on $E$ by

$$
\phi(\theta(\alpha) x)=\theta(\alpha)(x+\hat{u}(\alpha, x)) .
$$

Then because of condition (P) both $E \cup\{0\}$ and $\phi(E) \cup\{0\}$ are neighbourhoods of 0 in $D$. This concludes the proof.

A simple example will show the effect of assumption (ii) of Theorem 5.1. Let $X=l^{2}$ with

$$
g(x)=\sum_{0}^{\infty}\left((1 / n) x_{n}^{2}-x_{n}^{3}\right) \quad \text { and } \quad h(x)=\sum_{0}^{\infty}(1 / n) x_{n}^{2}
$$


where $x=\left(x_{n}\right)_{0}^{\infty}$. We take $\theta(\alpha)=\alpha I, \pi(\alpha)=\alpha^{2}$ and $S=\{x:\|x\|=1\}$. According to Theorem 2.1 there are $\theta$-sets $E_{1}$ and $E_{2}$ and a surjective homeomorphism $\phi: E_{1} \cup$ $\{0\} \rightarrow E_{2} \cup\{0\}$ such that $\phi(0)=0$ and $g(x)=h(\phi(x))$ for all $x \in E_{1} \cup\{0\}$. But $E_{1} \cup\{0\}$ cannot be a neighbourhood of 0 since $g$ takes negative values in every neighbourhood of 0 whereas $h$ is everywhere nonnegative. Of all the assumptions of Theorem 5.1 only (ii) is violated, since $\|D h(x)\|$ is not bounded away from 0 on $S$.

As in the above example whenever $X$ is a Hilbert space and $Y=\mathbf{R}$ condition (ii) is equivalent to requiring that $\|D h(x)\|$ should be bounded away from zero on the unit sphere. If $h$ is homogeneous of degree $d$ and $g$ is a $C^{d+1}$ mapping, we obtain a case of Kuiper's theorem [4, corollary on p. 202].

6. Extension of the main theorem: Nonlinear trajectories. A more farreaching extension of Theorem 2.1 is obtained by replacing $\theta(\alpha)$ by a nonlinear operator. The linearity of $\theta(\alpha)$ is not used in the proof so that the change is straightforward and produces a more general theorem. The notation $\theta(\alpha, x)$ will replace the notation $\theta(\alpha) x$ of Theorem 2.1. We suppose that on an open set $N$ such that $0 \in \bar{N} \backslash N$ there is a $C^{\infty}$ local flow $\Gamma^{t}$ such that $\Gamma^{t}(x)$ is defined for all $x \in N$ and $t \geq 0$, and $\lim _{t \rightarrow \infty} \Gamma^{t}(x)=0$ locally uniformly in $x$. Then we set $\theta(\alpha, x)=\Gamma^{-\log \alpha}(x)$ whenever the right-hand side is defined.

The relations $(2.1)$ and $(2.2)$ become

$$
\begin{gathered}
\lim _{\alpha \rightarrow 0+} \pi\left(\alpha^{-1}\right) g(\theta(\alpha, x))=h(x), \\
\lim _{\alpha \rightarrow 0+} \pi\left(\alpha^{-1}\right) D g(\theta(\alpha, x)) D_{2} \theta(\alpha, x)=D h(x),
\end{gathered}
$$

where the limits are locally uniform for $x \in N$.

It is the author's belief that by using nonlinear trajectories it is possible to unify Theorem 2.1 with the results of Kuiper and Kuo on $C^{0}$-sufficiency of jets. The problem is to determine the trajectories so that (6.1) and (more difficult) (6.2) are satisfied when $g$ and $h$ realise the same jet and the Kuiper-Kuo conditions hold. For the present we stick to applications in which the trajectories are fairly obvious.

We shall show how nonlinear trajectories can be used to study bifurcation when the Fredholm condition fails, and obtain branching results similar to those in [6].

We assume that $g: X \rightarrow Y$ is a $C^{n}$ mapping such that $g(0)=0$, and we denote ker $D g(0)$ by $V$ and $\operatorname{ran} D g(0)$ by $W$. We suppose that $\operatorname{dim} V<\infty$, that $\operatorname{codim} \bar{W}<$ $\infty$, and that $W$ is not closed, so that $D g(0)$ is not a Fredholm operator. We choose topological complements $Z$ and $U$ so that $X=V \oplus Z$ and $Y=\bar{W} \oplus U$. We denote the restrictions $D g(0) \mid Z$ and $D^{2} g(0)(x, \cdot) \mid Z$ by $T_{0}$ and $L(x)$ respectively. Note that $L(x)$ is a linear operator from $Z$ to $Y$ which depends linearly on $x$.

Consider the open set $K$ of all points $v$ in $V$ such that the range of $T_{0}+L(v)$ is a topological complement of $U$. We note that $0 \notin K$ precisely because $D g(0)$ is not a Fredholm operator. However $K$ may contain points arbitrarily near to 0 , and it is this possibility which we shall exploit instead of the Fredholm condition.

We assume in fact that there is an open cone $K_{0}$ in $K$ with vertex at 0 . Let $P: Y \rightarrow U$ be projection with kernel $\bar{W}$. We make the following assumption concerning $K_{0}$.

$\left(\mathrm{C}_{1}\right) \quad$ For each $v \in K_{0}$ and $y \in \bar{W}, \lim _{\alpha \rightarrow 0+} \alpha\left((I-P)\left(T_{0}+L(\alpha v)\right)\right)^{-1} y=0$. 
Note that for $0<\alpha<1$ the operator $(I-P)\left(T_{0}+L(\alpha v)\right)$ is an isomorphism of $Z$ onto $\bar{W}$ which approaches the singular $T_{0}$ as $\alpha$ approaches 0 . The condition $\left(\mathrm{C}_{1}\right)$ limits the growth of its inverse as $\alpha \rightarrow 0$, but only by requiring strong convergence as opposed to norm convergence. It is easily seen that convergence in $\left(C_{1}\right)$ is locally uniform in $y$ and $v$.

For each $v \in K_{0}$ let $J(v)$ be the projection from $Y$ onto the range of $T_{0}+L(v)$ with kernel $U$. We can write

$$
J(v)=\left(T_{0}+L(v)\right)\left((I-P)\left(T_{0}+L(v)\right)\right)^{-1}(I-P)
$$

which shows that $J(v)$ depends smoothly on $v$. We impose the following condition.

$$
\begin{aligned}
& \lim _{\alpha \rightarrow 0+} P J(\alpha v)=0 \text { for each } v \in K_{0} \text { where the limit is in the } \\
& \text { norm topology. }
\end{aligned}
$$

We shall point out later some instances of conditions $\left(C_{1}\right)$ and $\left(C_{2}\right)$, but first a theorem, analogous to Theorem 1 of $[6]$.

THEOREM 6.1. Let $B: V \rightarrow U$ be the mapping $B(v)=P D^{2} g(0) v^{(2)}$.

Suppose that $K_{0}$ satisfies $\left(\mathrm{C}_{1}\right)$ and $\left(\mathrm{C}_{2}\right)$ and that all solutions of $B(v)=0$ which lie in $K_{0}$ are regular ( $v$ is regular if $D B(v)$ is surjective). Let $N=\{0\} \cup$ $\left\{v \in K_{0}: B(v)=0\right\}$. Then there exists a neighbourhood $N_{0}$ of $\{0\}$ in $N$ and $a$ homeomorphism $F$ from $N_{0}$ onto a subset $M$ of $g^{-1}(0)$ such that

(i) $F(0)=0$;

(ii) the restriction of $F$ to $N_{0} \backslash\{0\}$ is a diffeomorphism onto $M \backslash\{0\}$ and the latter is a $C^{n}$ manifold;

(iii) for each $v \in N_{0} \backslash\{0\}$ we can write $F(\alpha v)=\alpha v+o(\alpha)$ for $0<\alpha<1$.

PROOF. We define our trajectories in the set

$$
K_{0}+Z=\left\{x \in X: x=v+z, v \in K_{0}, z \in Z\right\} .
$$

We shall use the convention that the letters $x, v, z$ refer to elements of $X, V, Z$ respectively such that $x=v+z$, and that the same should be understood if subscripts, bars, primes etc. are attached to these letters. The trajectories are given by $\theta(\alpha, x)=x_{\alpha}=v_{\alpha}+z_{\alpha}$, where $v_{\alpha}=\alpha v$ and

$$
z_{\alpha}=\alpha^{2}\left(T_{0}+L(\alpha v)\right)^{-1} J(\alpha v)\left(T_{0}+L(v)\right) z=\alpha^{2} S(\alpha v)^{-1} S(v) z
$$

where we have written $S(v)$ for $(I-P)\left(T_{0}+L(v)\right)$. We leave it to the reader to check that $x_{\alpha \beta}=\left(x_{\alpha}\right)_{\beta}$. The main thing to note is that $J(\alpha \beta v) J(\alpha v)=J(\alpha \beta v)$. Furthermore $\lim _{\alpha \rightarrow 0+} x_{\alpha}=0$. This follows from condition $\left(\mathrm{C}_{1}\right)$.

The function $h$ of Theorem 2.1 is defined by

$$
h(x)=\frac{1}{2} D^{2} g(0) v^{(2)}+(I-P)\left(T_{0}+L(v)\right) z .
$$

The assumptions of the theorem and the definition of $K$ imply that $D h(x)$ is surjective whenever $h(x)=0$ and $x \in K_{0}+Z$. The idea is to apply Theorem 2.1 on the set

$$
D=\left\{x \in K_{0}+Z: D h(x) \text { is surjective }\right\} .
$$

Then $D$ is open, $\theta$-invariant, contains all zeros of $h$ in $K_{0}+Z$ and $h \mid D$ is a submersion. We defer the checking of (6.1) and (6.2) to observe how the conclusions 
of the theorem are reached. We obtain a diffeomorphism $\phi$ defined on a $\theta$-set $E$ of $D$ and having the property $g(\phi(x))=h(x)$ for all $x \in E$.

The zero-set of $h$ in $D$ and the zero-set of $B$ in $K_{0}$ (which is just $N \backslash\{0\}$ ) are closely related. The former is the image of the latter under the mapping $\psi$ given by

$$
\psi(v)=v-\frac{1}{2} S(v)^{-1} D^{2} g(0) v^{(2)} .
$$

We note that $\psi$ maps the curves $\alpha \mapsto \alpha v$ to $\theta$-trajectories and preserves tangents at the origin. In fact

$$
\begin{aligned}
\psi(\alpha v) & =\alpha v-\frac{1}{2} S(\alpha v)^{-1} D^{2} g(0)(\alpha v)^{(2)} \\
& =\alpha v-\frac{1}{2} \alpha^{2} S(\alpha v)^{-1} S(v) S(v)^{-1} D^{2} g(0) v^{(2)}=\theta(\alpha, \psi(v)) .
\end{aligned}
$$

Since $N$ is locally compact the $\theta$-set $E$ contains all points of $\psi(N \backslash\{0\})$ sufficiently near to 0 , and so it contains $\psi\left(N_{0} \backslash\{0\}\right)$ for a suitable neighbourhood $N_{0}$ of 0 in $N$. We define $F$ to be the restriction of $\phi \circ \psi$ to $N_{0}$.

Finally we check relations (6.1) and (6.2) where $\pi(\alpha)=\alpha^{2}$. Firstly we note that $\alpha^{-1} \theta(\alpha, x)$ is bounded as $\alpha \rightarrow 0$ and the bound is locally uniform with respect to $x$. In fact by condition $\left(\mathrm{C}_{1}\right)$ and the uniform boundedness principle there is a bound on the norm of $\alpha S(\alpha v)^{-1}$ for $0<\alpha<1$ which is locally uniform with respect to $v$ in $K_{0}$. We leave it to the reader to check that $\alpha^{-2} h\left(x_{\alpha}\right)=h(x)$ so that $h$ is $\theta$-homogeneous. In doing this note that $(I-P) J(\alpha v)=I-P$ for $v \in K_{0}$. Now we let $f(x)=g(x)-h(x)$. We can write

$$
g(x)=T_{0} z+\frac{1}{2} D^{2} g(0)(v+z)^{(2)}+R(x)
$$

where $R(x)=O\left(\|x\|^{3}\right)$ so that

$$
f(x)=P L(v) z+\frac{1}{2} D^{2} g(0) z^{(2)}+R(x) .
$$

We need to check that

$$
\lim _{\alpha \rightarrow 0+} \alpha^{-2} f(\theta(\alpha, x))=0
$$

and

$$
\lim _{\alpha \rightarrow 0+} \alpha^{-2} D f(\theta(\alpha, x)) D_{2} \theta(\alpha, x)=0
$$

locally uniformly for $x \in K_{0}+Z$. We take each part of $f$ separately. As regards (6.3)

$$
\begin{gathered}
\alpha^{-2} P L\left(v_{\alpha}\right) z_{\alpha}=P L(v)\left(\alpha S(\alpha v)^{-1}\right) S(v) z \rightarrow 0 \text { by }\left(\mathrm{C}_{1}\right) . \\
\alpha^{-2} \frac{1}{2} D^{2} g(0) z_{\alpha}^{(2)}=\frac{1}{2} D^{2} g(0)\left(\alpha S(\alpha v)^{-1} S(v) z\right)^{(2)} \rightarrow 0 \text { by }\left(\mathrm{C}_{1}\right) . \\
\left\|\alpha^{-2} R\left(x_{\alpha}\right)\right\| \leq \text { const }\left\|\alpha^{-1} x_{\alpha}\right\|^{2}\left\|x_{\alpha}\right\| \rightarrow 0 \text { since } \alpha^{-1} x_{\alpha} \text { is bounded. }
\end{gathered}
$$

Next we consider (6.4). We use the notation $D_{2} \theta(\alpha, x) \bar{x}=\bar{x}_{\alpha}=\bar{v}_{\alpha}+\bar{z}_{\alpha}$ although properly speaking $\bar{x}_{\alpha}$ depends on $\alpha, x$ and $\bar{x}$. Then $\bar{v}_{\alpha}=\alpha \bar{v}$ and

$$
\begin{aligned}
\bar{z}_{\alpha}= & \alpha^{2} S(\alpha v)^{-1} S(v) \bar{z}+\alpha^{2} S(\alpha v)^{-1}(I-P) L(\bar{v}) z \\
& -\alpha^{3} S(\alpha v)^{-1}(I-P) L(\alpha \bar{v}) S(\alpha v)^{-1} S(v) z .
\end{aligned}
$$


The first part of $\alpha^{-2} D f\left(x_{\alpha}\right) \bar{x}_{\alpha}$ is

$$
\alpha^{-2} P L(\alpha \bar{v}) z_{\alpha}+\alpha^{-2} P L(\alpha v) \bar{z}_{\alpha} .
$$

This expression defines a linear operator acting on $(\bar{v}, \bar{z})$ which should converge to 0 in the norm topology (i.e. uniformly for bounded $\bar{v}$ and $\bar{z}$ ). It is sufficient to check mere pointwise (i.e. strong) convergence in terms not involving $\bar{z}$ since $V$ is finite dimensional. This leaves only the term $\alpha P L(v) S(\alpha v)^{-1} S(v) \bar{z}$ unaccounted for by condition $\left(\mathrm{C}_{1}\right)$. We can rewrite it as $P J(\alpha v) S(v) \bar{z}$ and now condition $\left(\mathrm{C}_{2}\right)$ implies convergence to 0 in the norm topology.

The next term of $\alpha^{-2} D f\left(x_{\alpha}\right) \bar{x}_{\alpha}$ is $\alpha^{-2} D^{2} g(0)\left(z_{\alpha}, \bar{z}_{\alpha}\right)$. The norm of this is less than a constant times $\left\|\alpha^{-1} z_{\alpha}\right\| \cdot\left\|\alpha^{-1} \bar{z}_{\alpha}\right\|$. The first factor tends to 0 and the second is bounded by a constant times $\|\bar{x}\|$. Finally the term $\alpha^{-2} D R\left(x_{\alpha}\right) \bar{x}_{\alpha}$ is dealt with by writing $D R(x)=R_{1}(x) x^{(2)}$ where $R_{1}(x)$ is a bilinear mapping with values in $L(X, Y)$.

To conclude the proof we point out that the invariant subbundle of the tangent bundle of $K_{0}+Z$ is constructed as in remark (3.5).

We shall give a simple example where conditions $\left(C_{1}\right)$ and $\left(C_{2}\right)$ can be verified by exploiting the spectral properties of selfadjoint operators in a Hilbert space. Other examples can be found by considering spaces of almost periodic functions. So let $H$ be a Hilbert space and $A$ a bounded selfadjoint operator, such that $\operatorname{ker} A$ is one dimensional and $\operatorname{ran} A$ is not closed. We suppose that there is an interval $(-r, 0)$ with $r>0$, not lying in the spectrum of $A$. Consider the bifurcation problem $(A-\lambda I) x=f(x)$ where $f: H \rightarrow H$ is a $C^{\infty}$ mapping such that $f(0)=0$ and $D f(0)=0$. The problem of bifurcation at $(\lambda, x)=(0,0)$ is not covered by the usual theorem about bifurcation at simple eigenvalues because $\operatorname{ran} A$ is not closed.

We set $X=\mathbf{R} \times H, Y=H, g(\lambda, x)=(A-\lambda I) x-f(x)$. Translating from Theorem 6.1 we have: $V=\mathbf{R} \times \operatorname{ker} A ; U=\operatorname{ker} A ; W=\operatorname{ran} A ; Z=\{0\} \times$ $\overline{\operatorname{ran} A} ; P: H \rightarrow U$ is orthogonal projection. Let $t_{0}$ be a basis vector in ker $A$ and let $P D^{2} f(0) t_{0}^{(2)}=c t_{0}$. If we write $t \in \operatorname{ker} A$ as $\mu t_{0}$ for $\mu \in \mathbf{R}$ the equation $P D^{2} g(0) v^{(2)}=0$ becomes $\lambda \mu+\frac{1}{2} c \mu^{2}=0$.

The root-lines are $\mu=0$ and $\lambda+\frac{1}{2} c \mu=0$, and if $W$ were closed they would be the tangents at $(0,0)$ of solution-curves of $g(\lambda, x)=0$. However as $W$ is not closed the situation is more complicated, though $\mu=0$ still defines the trivial curve of solutions $x=0$.

For $v=\left(\lambda, \mu t_{0}\right) \in \mathbf{R} \times \operatorname{ker} A$ and $z=(0, x) \in\{0\} \times \overline{\operatorname{ran} A}$ we have, translating from Theorem 6.1

$$
\left(T_{0}+L(v)\right) z=A x-\lambda x-\mu D^{2} f(0)\left(t_{0}, x\right)=A x-\lambda x-\mu B x
$$

where we have written $B$ for $D^{2} f(0)\left(t_{0}, \cdot\right)$. Now $A-\lambda I$ is invertible if $-r<\lambda<0$, and $\left\|(A-\lambda I)^{-1}\right\|=\lambda^{-1}$ if $-\frac{1}{2} r<\lambda<0$. Thus $A-\lambda I-\mu B$ is invertible if $-\frac{1}{2} r<\lambda<0$ and $|\mu|<\|B\|^{-1}|\lambda|$. This defines a cone in $V$.

Let us suppose that the part of the line $\lambda+\frac{1}{2} c \mu=0$ with $\lambda<0$ falls inside the cone $|\mu|<\|B\|^{-1}|\lambda|$. Then we claim that it is tangent at $(0,0)$ to a curve of solutions of $g(\lambda, x)=0$ ending at $(0,0)$ and, of course, bifurcating from the trivial line.

We shall apply Theorem 6.1 using for $K_{0}$ a cone in $V$ which contains that part of the root-line $\lambda+\frac{1}{2} c \mu=0$ with $-\frac{1}{2} r<\lambda<0$ and lies in the region $|\mu|<$ 
$\|B\|^{-1}|\lambda|$. For $v \in K_{0}$ the operator $(I-P)\left(T_{0}+L(v)\right)$ is the restriction to $\overline{\operatorname{ran} A}$ of $A-\lambda I-\mu(I-P) B$. Its inverse may be written either as

$$
(A-\lambda I)^{-1}\left[I-\mu(I-P) B(A-\lambda I)^{-1}\right]^{-1}
$$

or as

$$
\left[I-\mu(A-\lambda I)^{-1}(I-P) B\right]^{-1}(A-\lambda I)^{-1}
$$

where all operators are restricted to $\overline{\operatorname{ran} A}$. Now $\left(C_{1}\right)$ is equivalent to the statement

$$
\lim _{\alpha \rightarrow 0+} \alpha\left[I-\alpha \mu(A-\alpha \lambda I)^{-1}(I-P) B\right]^{-1}(A-\alpha \lambda I)^{-1} y=0
$$

for all $y \in \overline{\operatorname{ran} A}$. This is valid because of a basic property of selfadjoint operators.

LEMMA. For all $y \in \overline{\operatorname{ran} A}, \lim _{\beta \rightarrow 0+} \beta(A+\beta I)^{-1} y=0$.

This establishes condition $\left(C_{1}\right)$ if we take into account the bound

$$
\left\|\left(I-\alpha \mu(A-\alpha \lambda I)^{-1}(I-P) B\right)^{-1}\right\| \leq 1 /\left(1-|\mu| \cdot|\lambda|^{-1}\|B\|\right)
$$

obtained from the usual expansion $(I-T)^{-1}=\sum_{n=0}^{\infty} T^{n}$.

As no reference for the lemma could be found we shall indicate its proof. Let $E$ be the projection-valued measure associated with the spectral resolution of $A$. Then

$$
\beta(A+\beta I)^{-1} y=\int \beta(\lambda+\beta)^{-1} d E_{y}(\lambda)
$$

where $E_{y}$ is the vector-valued measure $E(\cdot) y$. Now $\lim _{\beta \rightarrow 0+} \beta(\lambda+\beta)^{-1}=0$ for all $\lambda$ except $\lambda=0$. But $E(\{0\}) y=0$ since $y \in(\operatorname{ker} A)^{\perp}$. Thus $\lim _{\beta \rightarrow 0+} \beta(\lambda+\beta)^{-1}=0$ almost everywhere with respect to $E_{y}$ and is uniformly bounded on the spectrum of $A$. The result then follows from the dominated convergence theorem.

Finally we must check condition $\left(\mathrm{C}_{2}\right)$. We have that

$$
P J(\alpha v)=-\alpha \mu P B(A-\alpha \lambda I)^{-1}\left[I-\alpha \mu(I-P) B(A-\alpha \lambda I)^{-1}\right]^{-1}(I-P) .
$$

The conclusion will follow if we show that $\left\|\alpha P B(A-\alpha \lambda I)^{-1}\right\| \rightarrow 0$ as $\alpha \rightarrow 0$. This follows from an elementary property of selfadjoint operators for which again no reference could be found.

LEMMA. Let $\left\{A_{i}\right\}$ be a generalised sequence of selfadjoint operators which converges strongly to 0 . Then if $C$ is an operator of finite rank, $\left\{C A_{i}\right\}$ converges to 0 in norm.

PROOF. There are vectors $x_{1}, \ldots, x_{m}$ and $c_{1}, \ldots, c_{m}$ such that

$$
C x=\sum_{j=1}^{m}\left\langle x, c_{j}\right\rangle x_{j} .
$$

Then

$$
C A_{i} x=\sum_{j=1}^{m}\left\langle A_{i} x, c_{j}\right\rangle x_{j}=\sum_{j=1}^{m}\left\langle x, A_{i} c_{j}\right\rangle x_{j}
$$

Thus

$$
\left\|C A_{i}\right\| \leq \sum_{j=1}^{m}\left\|A_{i} c_{j}\right\|\left\|x_{j}\right\| .
$$


Since $\lim _{i}\left\|A_{i} c_{j}\right\|=0$ for each $j$ we have $\lim _{i}\left\|C A_{i}\right\|=0$.

REMARK. There is a further generalisation of Theorem 2.1, obtained by assuming that the limit-set of the trajectories of $\theta$ is a set other than the origin. It could, for example, be a curve or surface. The invariant set $D$ must be in the complement of the limit-set. Since no property of the limit-set is used in the proof, the theorem can be carried over without change. In this connection it is appropriate to mention the work of Shearer [10], in which the zero-set of $g$ is assumed to contain a curve of critical points through the origin.

7. The method of scaling. If Theorem 2.1 is used to compare the zerosets near 0 of $g$ and $h$, a severe problem arises. The diffeomorphism $\phi$ may have no differentiable extension to the point 0 . This makes it difficult to compare "tangents" to the zero-sets at 0 itself.

The mapping $\phi^{-1}$, which maps $h^{-1}(0)$ to $g^{-1}(0)$, is most easily envisaged on trajectories, for

$$
\phi^{-1}(\theta(\alpha) x)=\theta(\alpha)(x+\eta(\alpha, x))
$$

where $\lim _{\alpha \rightarrow 0+} \eta(\alpha, x)=0$ locally uniformly in $x$. We might expect that the trajectory and its image have the same tangent at 0 .

Tangents to trajectories exist at the origin when the trajectories form a node. We shall specialise further and assume that $X$ is a direct sum of $\theta$-invariant subspaces $X_{1}, \ldots, X_{p}$ and on $X_{i}$ the operator $\theta(\alpha)$ is multiplication by $\alpha^{k_{i}}$ where $k_{1}, \ldots, k_{p}$ are all positive. We refer to such a $\theta(\alpha)$ as a simple scaling. We may assume that $k_{1}<k_{2}<\cdots<k_{p}$. If $\sigma=k_{p}-k_{1}$ and

$$
\pi\left(\alpha^{-1}\right) g(\theta(\alpha) x)-h(x)=o\left(\alpha^{\sigma}\right)
$$

locally uniformly for $x \neq 0$ then $\phi^{-1}$ preserves the tangent of every trajectory. In fact by the results of $\S 4$, the restriction of $\phi$ to any finite-dimensional $\theta$-invariant submanifold containing 0 has derivative the identity at 0 . In practice this often fails because the higher-order terms do not vanish so fast. We then have little choice but to think of $h^{-1}(0)$ as the union of trajectories, and examine the image of each under $\phi^{-1}$.

Identify $X$ with the direct product $X_{1} \times X_{2} \times \cdots \times X_{p}$. The trajectory of $\left(x_{1}, x_{2}, \ldots, x_{p}\right)$ is the curve $\alpha \mapsto\left(\alpha^{k_{1}} x_{1}, \ldots, \alpha^{k_{p}} x_{p}\right)$ defined for $\alpha>0$. This is tangent to $\left(x_{1}, 0, \ldots, 0\right)$ at 0 provided $x_{1} \neq 0$. Its image under $\phi^{-1}$ has the form

$$
\alpha \mapsto\left(\alpha^{k_{1}}\left(x_{1}+\eta_{1}(\alpha)\right), \ldots, \alpha^{k_{p}}\left(x_{p}+\eta_{p}(\alpha)\right)\right)
$$

where $\eta_{i}(0)=0$ for each $i$. This curve is also tangent to $\left(x_{1}, 0, \ldots, 0\right)$ provided $x_{1} \neq 0$. Those trajectories through points such that $x_{1}=0$ we shall refer to as exceptional. Thus $\phi^{-1}$ preserves the tangents at 0 of all nonexceptional trajectories.

From the form of $\phi^{-1}$ on trajectories we can assert the following: if $W$ is a finite-dimensional submanifold of $X_{1} \times\{0\} \times \cdots \times\{0\}$ which contains 0 , then the restriction of $\phi^{-1}$ to $W$ has derivative the identity at 0 .

If $\left(x_{1}, \ldots, x_{p}\right)$ is such that no $x_{i}$ is 0 then the tangent at 0 of the projection of the trajectory into each space $X_{i} \times \cdots \times X_{p}(i=1, \ldots, p)$ is preserved.

On the other hand it is not clear whether the image of an exceptional trajectory even possesses a tangent at 0 , let alone whether it is preserved. Here it is of interest to consider the situation described in remark (3.9). The assumptions are that:

(i) $g$ is $C^{k+m}$ on a neighbourhood of 0 . 
(ii) $\theta$ is $C^{k+m}$ on $[0, \infty)$.

(iii) $\pi\left(\alpha^{-1}\right)=\alpha^{-k} \pi_{1}(\alpha)$ where $\pi_{1}$ is $C^{k+m}$ on $[0, \infty)$.

These imply that the mappings $\eta_{i}(\alpha)$ are $C^{m}$ for $\alpha \geq 0$, and we repeat that they satisfy $\eta_{i}(0)=0$.

PROPOSITION 7.1. If $m \geq k_{p}-k_{1}$ then the image of every trajectory under $\phi^{-1}$ has a tangent at 0 .

PROOF. Consider the trajectory through $\left(x_{1}, \ldots, x_{p}\right)$. For each $j$ let $r_{j}$ be the greatest integer $r$ such that $x_{j}+\eta_{j}(\alpha)=O\left(\alpha^{r}\right)$ as $\alpha \rightarrow 0$. If there is no greatest integer we set $r_{j}=\infty$. Choose a subscript $q$ such that $x_{q} \neq 0$. Then $r_{q}=0$. Hence if $\mu=\min _{j}\left(k_{j}+r_{j}\right)$ then $\mu \leq k_{q}$. The image of the trajectory will have a tangent at 0 if for all $j$ such that $k_{j}+r_{j}=\mu$ the limit

$$
\lambda_{j}=\lim _{\alpha \rightarrow 0+} \alpha^{-r_{j}}\left(x_{j}+\eta_{j}(\alpha)\right)
$$

exists and for at least one such $j$ it is not 0 . Now assume that $m \geq k_{p}-k_{1}$. If $k_{j}+r_{j}=\mu$ then $r_{j}=\mu-k_{j} \leq k_{q}-k_{j} \leq m$. Since each $\eta_{j}$ is of class $C^{m}$ for $\alpha \geq 0$ the limits $\lambda_{j}$ all exist. Suppose that all such $\lambda_{j}$ are 0 . Then we must have $r_{j}=m$ whenever $k_{j}+r_{j}=\mu$. If, however, $k_{j}+r_{j}=\mu$ we have

$$
k_{q} \geq \mu=k_{j}+r_{j}=k_{j}+m \geq k_{j}+k_{p}-k_{1} \geq k_{p} \text {. }
$$

Hence $k_{q}=\mu=k_{p}$ so that $\mu$ is also attained at $q$ where $r_{q}=0$ and $\lambda_{q}=x_{q} \neq 0$, a contradiction. This concludes the proof.

We can interpret Proposition 7.1 as saying that the nodal structure of $h^{-1}(0)$ is preserved, though not necessarily the actual tangents of exceptional trajectories.

In order to illustrate the determination of bifurcation sets we shall consider the example treated by Chow, Hale and Mallet-Paret [2]. Let $M: \mathbf{R}^{N} \rightarrow \mathbf{R}^{N}$ be a homogeneous cubic polynomial, $L: \mathbf{R}^{N} \rightarrow \mathbf{R}^{N}$ a linear operator, and $v \in \mathbf{R}^{N}$ a fixed vector other than 0 . We consider the equation

$$
f\left(u, \lambda_{1}, \lambda_{2}\right)=M(u)+\lambda_{1} L u+\lambda_{2} v+r\left(u, \lambda_{1}, \lambda_{2}\right)=0
$$

where $r$ is (for the present) a $C^{1}$ mapping, thought of as "higher-order terms". To apply Theorem 2.1 we define

$$
\begin{aligned}
X & =\mathbf{R}^{N} \times \mathbf{R} \times \mathbf{R}, \quad x=\left(u, \lambda_{1}, \lambda_{2}\right), \\
g(x) & =f\left(u, \lambda_{1}, \lambda_{2}\right), \quad h(x)=M(u)+\lambda_{1} L u+\lambda_{2} v, \\
\theta(\alpha) x & =\left(\alpha u, \alpha^{2} \lambda_{1}, \alpha^{3} \lambda_{2}\right), \quad \pi(\alpha)=\alpha^{3} .
\end{aligned}
$$

Relations (2.1) and (2.2) will be satisfied if

$$
\lim _{\alpha \rightarrow 0+} \alpha^{-2} \operatorname{Dr}\left(\alpha u, \alpha^{2} \lambda_{1}, \alpha^{3} \lambda_{2}\right) \cdot\left[\begin{array}{ccc}
1 & 0 & 0 \\
0 & \alpha & 0 \\
0 & 0 & \alpha^{2}
\end{array}\right]=0
$$

uniformly for bounded $\left(u, \lambda_{1}, \lambda_{2}\right)$. If $r$ is $C^{4}$ we can assume more simply (see remark (3.8)) that

$$
r\left(u, \lambda_{1}, \lambda_{2}\right)=o\left(|u|^{3}+\left|\lambda_{1}\right|^{3 / 2}+\left|\lambda_{2}\right|\right) .
$$




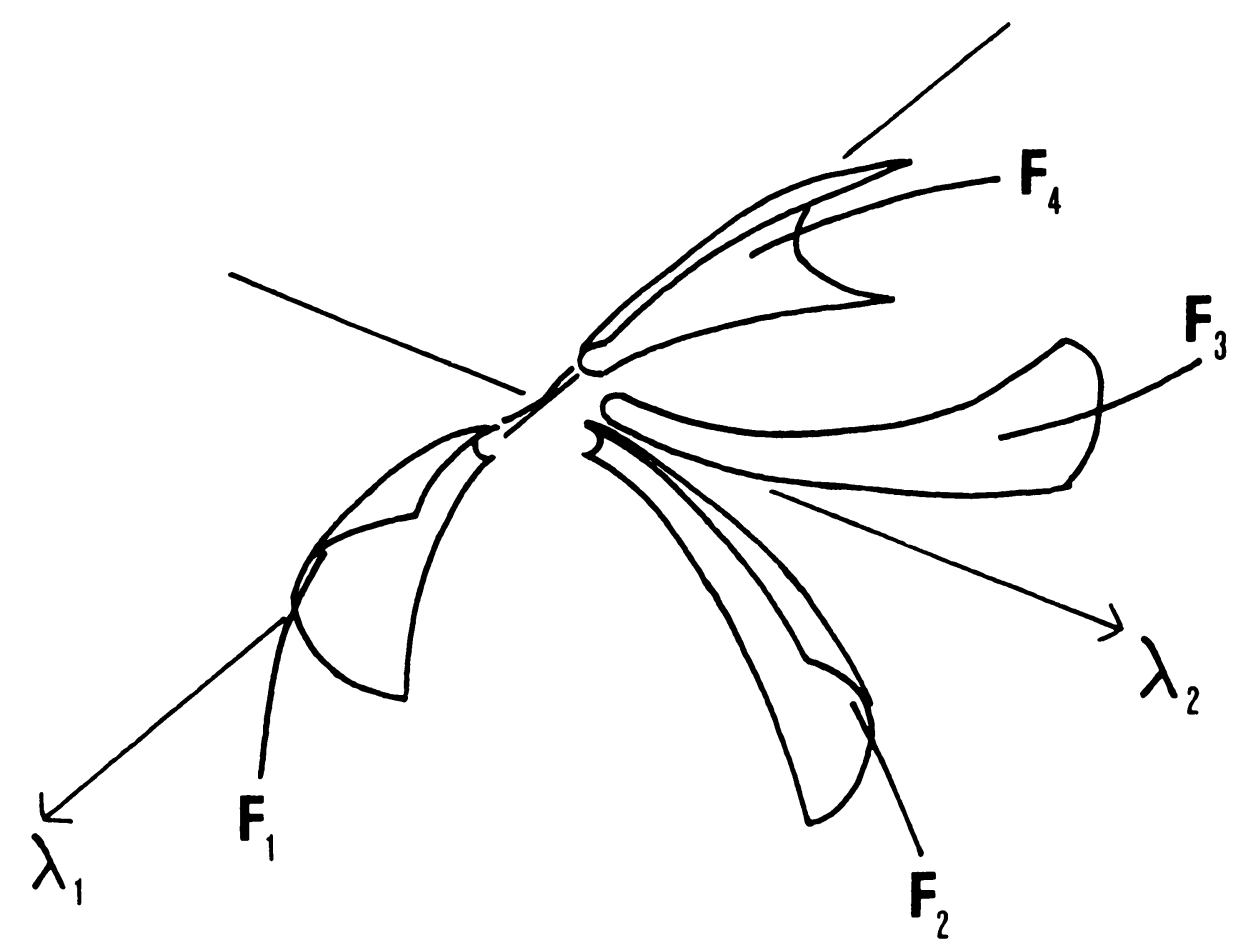

FIGURE 1

To use Theorem 2.1 it remains to check that $h$ is regular on its zero-set except at 0 . This is frequently equivalent to checking that certain curves, surfaces etc. meet transversally.

If $h$ is regular on its zero-set the latter will be a smooth manifold in general singular at 0 , and composed of trajectories. All these trajectories except the pair through $(0, \pm 1,0)$ lie in the region $u \neq 0$. They are tangent to a vector in $\mathbf{R}^{N} \times\{0\} \times$ $\{0\}$ and the same is true of their images in $g^{-1}(0)$. The two exceptional trajectories are mapped to curves with definite tangents at the origin if $g$ is $C^{5}$, according to Proposition 7.1. We can ensure preservation of all tangents at 0 of curves in $h^{-1}(0)$ if $D \phi(0)=I$ and this occurs if $r\left(\alpha u, \alpha^{2} \lambda_{1}, \alpha^{3} \lambda_{2}\right)=o\left(\alpha^{5}\right)$, according to $\S 4$.

The manifolds $h^{-1}(0)$ and $g^{-1}(0)$ will in general have critical points with respect to the projection $\left(u, \lambda_{1}, \lambda_{2}\right) \mapsto\left(\lambda_{1}, \lambda_{2}\right)$. The images of these points in the $\left(\lambda_{1}, \lambda_{2}\right)$-plane for the respective manifolds form the bifurcation sets for the problems $h\left(u, \lambda_{1}, \lambda_{2}\right)=0$ and $g\left(u, \lambda_{1}, \lambda_{2}\right)=0$ when $\lambda_{1}$ and $\lambda_{2}$ are regarded as parameters. The diffeomorphism $\phi^{-1}$ will not in general preserve the property of being a critical point for projection, and so it will not relate the two bifurcation sets.

Analytically, critical points for projection on $g^{-1}(0)$ are given by $g(x)=0$, $\operatorname{det} D_{u} g(x)=0$. We define mappings $G$ and $H$ from $\mathbf{R}^{N} \times \mathbf{R} \times \mathbf{R}$ to $\mathbf{R}^{N} \times \mathbf{R}$ by

$$
\begin{aligned}
& G\left(u, \lambda_{1}, \lambda_{2}\right)=\left(g(x), \operatorname{det} D_{u} g(x)\right), \\
& H\left(u, \lambda_{1}, \lambda_{2}\right)=\left(h(x), \operatorname{det} D_{u} h(x)\right) .
\end{aligned}
$$

We define $\pi(\alpha): \mathbf{R}^{N} \times \mathbf{R} \rightarrow \mathbf{R}^{N} \times \mathbf{R}$ by $\pi(\alpha)(u, t)=\left(\alpha^{3} u, \alpha^{2 N} t\right)$. It is now not too hard to verify that if the remainder term $r$ is $C^{5}$ and satisfies (*) then $G$ and $H$ satisfy relations (2.1) and (2.2). 


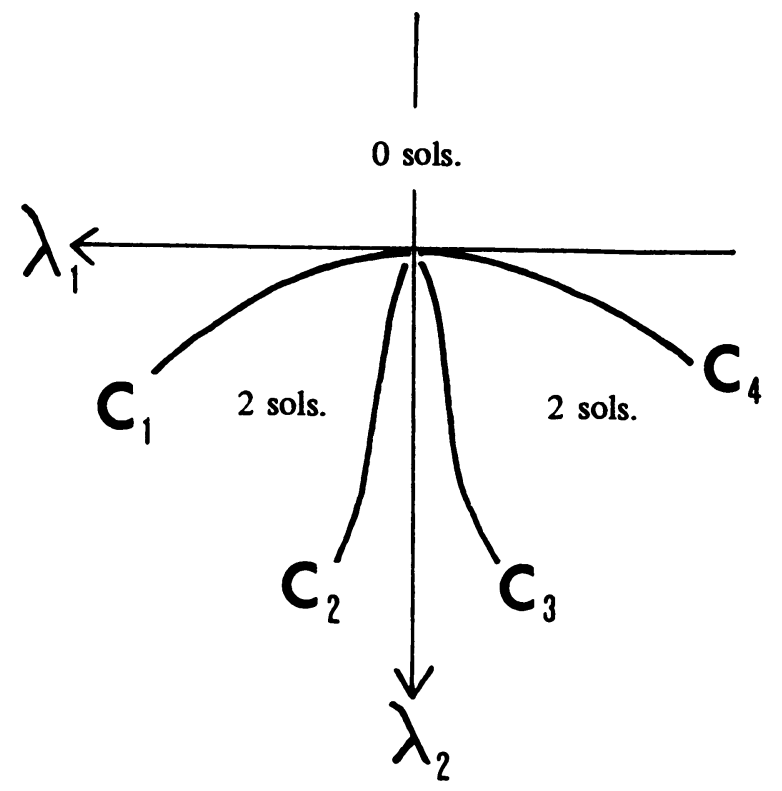

FIGURE 2

We now assume that $H$ is regular on its zero-set except at 0 . This assumption is slightly weaker than those used by Chow, Hale and Mallet-Paret [2, Theorem 4.1]. It implies that $h$ is regular on its zero-set (excepting, as always, the point 0 ).

The zero-set of $H$ consists of a finite number of trajectories, and none is exceptional, that is, they all have $u \neq 0$. Their images in $G^{-1}(0)$ have the same tangents at 0 , and projecting down to the $\left(\lambda_{1}, \lambda_{2}\right)$-plane we obtain the bifurcation set (see Figure 1). This consists of a finite number of curves, some, like $C_{1}$, tangent to $(1,0)$ at the origin, and others, like $C_{2}$, with indeterminate tangents, corresponding to trajectories in $H^{-1}(0)$ through points of the form $\left(u, 0, \lambda_{2}\right)$. These last curves were eliminated in [2] by assuming essentially that if $u \neq 0$ and $M(u)+\lambda_{2} v=0$ then $\operatorname{DM}(u)$ is nonsingular.

This example shows how Theorem 2.1 clarifies previously used hypotheses and can suggest new ones. However the important result proved in [2] that the number of solutions of $f\left(u, \lambda_{1}, \lambda_{2}\right)=0$ near to $(0,0,0)$ changes by two as $\left(\lambda_{1}, \lambda_{2}\right)$ crosses the bifurcation set does not fall out of the above calculations, and will be considered in the next section. Figure 1 shows parts of the surface

$$
\begin{gathered}
\frac{1}{2} x^{2}+\frac{1}{2} y^{2}-\lambda_{2}+\text { higher-order terms }=0 \\
x y-\lambda_{1} y-\lambda_{2}+\text { higher-order terms }=0
\end{gathered}
$$

in $\left(x, y, \lambda_{1}, \lambda_{2}\right)$-space. A quadratic polynomial was used, rather than a cubic, to simplify the calculations. There are four fold-lines, $F_{1}, F_{2}, F_{3}$ and $F_{4}$, with welldefined tangents at the origin independent of the higher-order terms. These lines are projected down to the curves $C_{1}, C_{2}, C_{3}$ and $C_{4}$ in the $\left(\lambda_{1}, \lambda_{2}\right)$-plane, shown in Figure 2. The tangents at the origin of $C_{1}$ and $C_{4}$ are well defined, but those of $C_{2}$ and $C_{3}$ depend on the higher-order terms. The number of solutions $(x, y)$ for 
each region of the $\left(\lambda_{1}, \lambda_{2}\right)$-plane may be calculated by dropping the higher-order terms, except between $C_{2}$ and $C_{3}$ where there may be none or four. The latter curves coincide with the positive $\lambda_{2}$-axis when the higher-order terms are zero, but the corresponding fold-lines remain always distinct. The geometry is most easily understood as a section of the "hyperbolic umbilic catastrophe", but the higher-order terms need not be a gradient. It is enough if they are $C^{3}$ and satisfy $\alpha^{-2} r\left(\alpha x, \alpha y, \alpha \lambda_{1}, \alpha^{2} \lambda_{2}\right) \rightarrow 0$ as $\alpha \rightarrow 0+$.

8. Two-parameter bifurcations. In this section we shall pursue further the problem of bifurcation sets motivated by the example in the last section. Let $g: \mathbf{R}^{N} \times \mathbf{R}^{2} \rightarrow \mathbf{R}^{N}$ be a $C^{n}$ mapping such that $g(0)=0$. We write $x=\left(u, \lambda_{1}, \lambda_{2}\right)$ for $x \in \mathbf{R}^{N} \times \mathbf{R}^{2}$ where $u \in \mathbf{R}^{2}$ and $\left(\lambda_{1}, \lambda_{2}\right) \in \mathbf{R}^{2}$. The point of view of this section is that the mappings $g_{\left(\lambda_{1}, \lambda_{2}\right)}: u \mapsto g\left(u, \lambda_{1}, \lambda_{2}\right)$ are deformations of the mapping $g_{(0.0)}$.

We are interested in the singular zero-set of $g$ near to 0 by which we mean the solutions of $g\left(u, \lambda_{1}, \lambda_{2}\right)=0$ near to 0 for which $D_{u} g\left(u, \lambda_{1}, \lambda_{2}\right)$ is singular. The projection of this set to $\left(\lambda_{1}, \lambda_{2}\right)$-space is the (local) bifurcation set.

Suppose that $\alpha \mapsto \theta(\alpha)$ is a multiplicative group of linear operators in $\mathbf{R}^{N} \times \mathbf{R}^{2}$ (as in $\S 2$ ) and that $h: \mathbf{R}^{N} \times \mathbf{R}^{2} \rightarrow \mathbf{R}^{N}$ is a $C^{n}$ mapping such that

$$
\lim _{\alpha \rightarrow 0+} \alpha^{-k} g(\theta(\alpha) x)=h(x)
$$

uniformly for $x$ in bounded sets. Under appropriate conditions, generalising those of $[\mathbf{2}]$, we shall relate the singular zero-set of $g$ to that of $h$, and it will consist of simple fold-points, a term which we proceed to define.

Let $f: \mathbf{R}^{N} \times \mathbf{R}^{p} \rightarrow \mathbf{R}^{N}$ be a $C^{2}$ mapping, which we regard as a $p$-parameter family of mappings from $\mathbf{R}^{N}$ into itself. Write $x=(u, \lambda)$ where $x \in \mathbf{R}^{N} \times \mathbf{R}^{p}, u \in \mathbf{R}^{N}$ and $\lambda \in \mathbf{R}^{p}$ and write $f_{\lambda}(u)=f(u, \lambda)$. A point $\left(u_{0}, \lambda_{0}\right)$ is said to be a simple fold-point if the following conditions hold.

(i) $f\left(u_{0}, \lambda_{0}\right)=0$.

(ii) $D_{1} f\left(u_{0}, \lambda_{0}\right)$ has rank $N-1$.

(iii) If $v$ spans $\operatorname{ker} D_{1} f\left(u_{0}, \lambda_{0}\right)$ then $D_{1}^{2} f\left(u_{0}, \lambda_{0}\right) v^{(2)} \notin \operatorname{ran} D_{1} f\left(u_{0}, \lambda_{0}\right)$.

(iv) $\operatorname{Ran} D_{2} f\left(u_{0}, \lambda_{0}\right) \not \subset \operatorname{ran} D_{1} f\left(u_{0}, \lambda_{0}\right)$.

If these four conditions hold it may be shown that there is a hypersurface $S$ in $\mathbf{R}^{p}$ containing $\lambda_{0}$, and neighbourhoods $U$ of $u_{0}$ in $\mathbf{R}^{N}$ and $W$ of $\lambda_{0}$ in $\mathbf{R}^{p}$, such that $S$ separates $W$ into three disjoint regions $W=W_{1} \cup W_{2} \cup(W \cap S)$ such that $\left(f_{\lambda} \mid U\right)^{-1}(0)$ is empty for $\lambda \in W_{1}$, consists of two points for $\lambda \in W_{2}$, and consists of one point for $\lambda \in W \cap S$. This is proved in [2]. It is an easy consequence of the Liapunov-Schmidt procedure and the Morse lemma.

THEOREM 8.1. Let $H: \mathbf{R}^{N} \times \mathbf{R}^{2} \rightarrow \mathbf{R}^{N} \times \mathbf{R}^{1}$ be defined by

$$
H(x)=\left(h(x), \operatorname{det} D_{u} h(x)\right) \text {. }
$$

Assume that:

(i) $\theta$ is $C^{n}$ on $[0, \infty)$.

(ii) $n-k \geq 2$.

(iii) $h(u, 0,0)=0$ if and only if $u=0$.

(iv) $H$ is regular on its zero-set except at 0. 
(v) There is a flow $\alpha \mapsto \psi(\alpha)$ in $\mathbf{R}^{2}$ (having the properties (i) and (ii) given for $\theta(\alpha)$ in §2) such that $\psi(\alpha) \circ F=F \circ \theta(\alpha)$, where $F: \mathbf{R}^{N} \times \mathbf{R}^{2} \rightarrow \mathbf{R}^{2}$ is projection. (In short, $\theta(\alpha)$ is a "lifting" of $\psi(\alpha)$.)

Then there is a neighbourhood $W$ of 0 in $\mathbf{R}^{N} \times \mathbf{R}^{2}$ such that the singular zero-set of $g$ in $W$ consists of a finite number of curves of simple fold-points. If $x_{1}, \ldots, x_{r}$ are points such that the zero-set of $H$ consists of the trajectories $\alpha \mapsto \theta(\alpha) x_{j}, j=$ $1, \ldots, r$, then the singular zero-set of $g \mid W$ consists of $r$ curves of the form

$$
\alpha \mapsto \theta(\alpha)\left(x_{j}+\eta_{j}(\alpha)\right)
$$

where $\lim _{\alpha \rightarrow 0+} \eta_{j}(\alpha)=0$. The mappings $\eta_{j}$ are of class $C^{n-k-1}$ on $[0, \infty)$.

PRoof. We define a mapping $G: \mathbf{R}^{N} \times \mathbf{R}^{2} \rightarrow \mathbf{R}^{N} \times \mathbf{R}^{1}$ by

$$
G(x)=\left(g(x), \operatorname{det} D_{u} g(x)\right) .
$$

The zeros of $G$ constitute the singular zero-set of $g$. If $\pi(\alpha): \mathbf{R}^{N} \times \mathbf{R}^{1} \rightarrow \mathbf{R}^{N} \times \mathbf{R}^{1}$ is defined by

$$
\pi(\alpha)(v, t)=\left(\alpha^{k} v, \alpha^{k N}\left(\operatorname{det} \bar{\theta}\left(\alpha^{-1}\right)\right) t\right)
$$

where $\bar{\theta}(\alpha)$ is the restriction of $\theta(\alpha)$ to $\mathbf{R}^{N} \times\{(0,0)\}$, then

$$
\lim _{\alpha \rightarrow 0+} \pi\left(\alpha^{-1}\right) G(\theta(\alpha) x)=H(x) \text {. }
$$

Furthermore because of conditions (i) and (ii) the mapping

$$
(\alpha, x) \mapsto \begin{cases}\pi\left(\alpha^{-1}\right) G(\theta(\alpha) x), & \alpha \neq 0, \\ H(x), & \alpha=0\end{cases}
$$

is of class $C^{n-k-1}$ on $[0, \infty) \times \mathbf{R}^{N+2}$. Applying Theorem 2.1 we get all the stated conclusions except that the singular zero-set of $g$ consists of simple-fold points in a neighbourhood $W$ of 0 .

If $G(x)=0, x \neq 0$ and $\|x\|$ is sufficiently small then $D G(x)$ is surjective. Consider such a point $x_{0}$ and let $u_{0}$ be a nonzero vector in the kernel of $D_{u} g\left(x_{0}\right)$. We may assume that

$$
x_{0}=\theta\left(\alpha_{0}\right)\left(x_{j}+\eta_{j}\left(\alpha_{0}\right)\right)
$$

for some $j$ and $\alpha_{0}>0$. The tangent-vector $v_{0}$ to the curve

$$
\alpha \mapsto \theta(\alpha)\left(x_{j}+\eta_{j}(\alpha)\right)
$$

at $x_{0}$ lies in the kernel of $D G\left(x_{0}\right)$ and so spans it since this space is one dimensional. Now $D g\left(x_{0}\right) \cdot\left(u_{0}, 0,0\right)=0$. We shall show that $W$ may be chosen so that the tangent-vectors to the curves of zeros of $G$ in $W \backslash\{0\}$ do not lie in $\mathbf{R}^{N} \times\{(0,0)\}$. Assuming this it follows that $\left(u_{0}, 0,0\right) \notin \operatorname{ker} D G\left(x_{0}\right)$, so that

$$
D(\operatorname{det})\left(D_{u} g\left(x_{0}\right)\right)\left(D_{u}^{2} g\left(x_{0}\right) u_{0}\right) \neq 0 \text {. }
$$

This is the same as

$$
(d / d t)_{t=0} \operatorname{det}\left[D_{u} g\left(x_{0}\right)+t D_{u}^{2} g\left(x_{0}\right) u_{0}\right] \neq 0
$$

and this implies that $\operatorname{ker} D_{u} g\left(x_{0}\right)$ is one dimensional and that $D_{u}^{2} g\left(x_{0}\right) u_{0}^{(2)} \notin$ $\operatorname{ran} D_{u} g\left(x_{0}\right)$ according to the following lemma. 
LEMMA. Let $T_{1}$ and $T_{2}$ belong to $L\left(\mathbf{R}^{N}, \mathbf{R}^{N}\right)$ and suppose that the function $\phi(t)=\operatorname{det}\left(T_{1}+t T_{2}\right)$ has a simple zero at $t=0$. Then $\operatorname{ker} T_{1}$ is one dimensional and $T_{2}\left(\operatorname{ker} T_{1}\right) \not \subset \operatorname{ran} T_{1}$.

PROOF. Let $\pi$ be a projection onto the kernel of $T_{1}$. Let

$$
A(t)=\left(T_{1}+t T_{2}\right)\left(t^{-1} \pi+I-\pi\right)=T_{1}+T_{2} \pi+t T_{2}(I-\pi) .
$$

Then $T_{1}+t T_{2}=A(t)(t \pi+I-\pi)$ and $\phi(t)=t^{r} \operatorname{det} A(t)$ where $r=\operatorname{dim} \operatorname{ker} T_{1}$. Hence $r=1$ and $\operatorname{det} A(0) \neq 0$. Thus $T_{1}+T_{2} \pi$ is nonsingular whence $\operatorname{ran}\left(T_{2} \pi\right) \not \subset \operatorname{ran} T_{1}$. This proves the lemma.

To conclude that $x_{0}$ is a simple fold-point of $g$ it remains to show that there exist $\lambda_{1}$ and $\lambda_{2}$ such that

$$
D g\left(x_{0}\right) \cdot\left(0, \lambda_{1}, \lambda_{2}\right) \notin \operatorname{ran} D_{u} g\left(x_{0}\right) .
$$

This follows from the facts that $D_{u} g\left(x_{0}\right)$ has rank $N-1$ and $D g\left(x_{0}\right)$ is surjective.

To complete the proof we must show that the tangents to the curves of zeros of $G$ do not lie in $\mathbf{R}^{N} \times\{(0,0)\}$ in a sufficiently small punctured neighbourhood of 0 . Consider the curve

$$
\alpha \mapsto \theta(\alpha)\left(x_{j}+\eta_{j}(\alpha)\right)
$$

The tangent is given by

$$
\begin{aligned}
v_{\alpha} & =\theta^{\prime}(\alpha)\left(x_{j}+\eta_{j}(\alpha)\right)+\theta(\alpha) \eta_{j}^{\prime}(\alpha) \\
& =\alpha^{-1} \theta(\alpha) B\left(x_{j}+\eta_{j}(\alpha)\right)+\theta(\alpha) \eta_{j}^{\prime}(\alpha)
\end{aligned}
$$

where $B=\theta^{\prime}(1)$. Using (v) we have that

$$
F v_{\alpha}=\alpha^{-1} \psi(\alpha) C\left(F x_{j}+F \eta_{j}(\alpha)\right)+\psi(\alpha) F \eta_{j}^{\prime}(\alpha)
$$

where $C=\psi^{\prime}(1)$. Hence $\lim _{\alpha \rightarrow 0+} \alpha \psi\left(\alpha^{-1}\right) F v_{\alpha}=C F x_{j}$. According to condition (iii) the right-hand side is not 0 . Hence for sufficiently small $\alpha$ we see that $F v_{\alpha} \neq 0$, that is, $v_{\alpha} \notin \mathbf{R}^{N} \times\{(0,0)\}$. This concludes the proof.

REMARKS. 1. The theorem is valid for nonlinear trajectories. The proof is unchanged except that the formula for $v_{\alpha}$ is more complicated.

2. The theorem does not hold if instead of (iv) we merely assume that $h$ is regular on its zero-set.

3. It would be interesting and useful to prove that under the hypotheses of Theorem 8.1 (but replacing $\mathbf{R}^{2}$ by $\mathbf{R}^{p}$ ) there was a diffeomorphism from the zeroset of $h$ to the zero-set of $g$ in some neighbourhood of 0 , which was a lifting of a diffeomorphism in the parameter-space $\mathbf{R}^{p}$.

4. The hypothesis (iv) that $H$ is regular on its zero-set implies that if $h(x)=0$ then $D_{u} h(x)$ has rank at least $N-1$. This is a rather restrictive condition if $\mathbf{R}^{2}$ is replaced by $\mathbf{R}^{p}$. Is there a weaker transversality condition which implies the same conclusion as in Remark 3 ?

9. Appendix 1: The existence of a subbundle transversal to ker $D h$. It was asserted in remark (2.5) that if $X$ is finite dimensional and $h$ of class $C^{n+1}$ then (i) implies (ii) in Theorem 2.1. We shall now prove this. Identify $X$ with $\mathbf{R}^{m}$ equipped with the Euclidean inner-product. 
LEMMA. There exists a hyperellipsoid $S$ in $\mathbf{R}^{m}$ given by $S=\{x:\langle A x, x\rangle=1\}$ for some positive selfadjoint operator $A$, such that each trajectory of $\theta$ meets $S$ transversally in a unique point.

PROOF. Let $\theta(\alpha)=e^{-(\log \alpha) B}$ where the spectrum of $B$ lies in the left half-plane. By a real linear transformation of coordinates, we may transform the matrix of $B$ to one that has real eigenvalues on the diagonal, blocks on the diagonal of the form $\left[\begin{array}{cc}\lambda & -\omega \\ \omega & \lambda\end{array}\right]$ corresponding to complex eigenvalues $\lambda \pm i \omega$, and all other entries less than $\varepsilon$ in absolute value for given $\varepsilon>0$. This well-known result was used (and proved) in a similar context in [11, p. 102]. We may therefore assume that $B$ has such a matrix in the standard coordinate system of $\mathbf{R}^{m}$. A simple calculation now shows that $\|x\|^{2}$ is strictly increasing on the trajectories if $\varepsilon$ is sufficiently small. Hence we may take $S$ to be the sphere $\|x\|^{2}=1$. For arbitrary $B$, therefore, $S$ is a hyperellipsoid of the stated form.

The same proof works incidentally in the nonlinear case where the trajectories of $\theta$ are the phase-curves of the differential equation $x^{\prime}=-B x+f(x)$ where $f(0)=0$ and $D f(0)=0$.

We can now prove remark (2.5). On the surface $S$ choose $F_{x}$ to be the orthogonal complement of $\operatorname{ker} D h(x)$. For $x \notin S$ define $F_{x}$ to be $\theta(\alpha) F_{y}$ where $y \in S$ and $\theta(\alpha) y=x$.

10. Appendix 2: Implicit functions. We collect together here various results on implicit functions, beginning with the implicit function theorem itself. For a concise proof see [14].

THEOREM 10.1. Let $F$ and $G$ be Banach spaces, $E$ a topological space, and $f$ a continuous mapping of an open subset $A$ of $E \times F$ into $G$ such that the derivative $D_{2} f$ exists and is continuous. Let $\left(x_{0}, y_{0}\right)$ be a point of $A$ such that $f\left(x_{0}, y_{0}\right)=0$ and $D_{2} f\left(x_{0}, y_{0}\right)$ is a linear homeomorphism of $F$ onto $G$. Then there are neighbourhoods $U$ of $x_{0}$ in $E$ and $V$ of $y_{0}$ in $F$, such that $U \times V \subset A$, and there exists a continuous function $v: U \rightarrow V$ such that $v\left(x_{0}\right)=y_{0}$ and $f(x, v(x))=0$ for all $x \in U$. Furthermore whenever $x \in U$ and $y \in V$ satisfy $f(x, y)=0$ then $y=v(x)$.

By slightly modifying Dieudonné's proof $[14$, p. 266, second paragraph] we can derive as a corollary an extremely convenient uniqueness criterion. The hypotheses and notation are those of Theorem 10.1 , but, be it noted, the neighbourhood $V$ is not mentioned.

COROLLARY 10.1. Let $S$ be a connected subset of $U \times F$ such that $f(x, y)=0$ for all $(x, y) \in S$ and suppose that $S$ contains a point of the form $(x, v(x))$. Then $y=v(x)$ for all $(x, y) \in S$.

This result (proved in [6]) is useful for patching together implicit functions. Let us assume that $E$ has a basis $U$ for its topology such that the intersection of any two members of $U$ is connected. Suppose that $P=\left\{\left(x_{0 i}, y_{0 i}\right): i \in I\right\}$ is a set of points, indexed by a set $I$, such that $f\left(x_{0 i}, y_{0 i}\right)=0$ and $D_{2} f\left(x_{0 i}, y_{0 i}\right)$ is invertible for each $i$. Then, applying the implicit function theorem at each point $\left(x_{0 i}, y_{0 i}\right)$, we can find a neighbourhood $U_{i}$ of $x_{0 i}$, such that $U_{i} \in \mathcal{U}$, and a continuous function $v_{i}: U_{i} \rightarrow F$ such that $f\left(x, v_{i}(x)\right)=0$ for all $x \in U_{i}$, and $y_{0 i}=v_{i}\left(x_{0 i}\right)$. Corollary 10.1 now implies that if there is a point $x$ in $U_{i} \cap U_{j}$ such that $v_{i}(x)=v_{j}(x)$ then equality 
holds for all $x$ in $U_{i} \cap U_{j}$. Under this condition we obtain an implicit function in a neighbourhood of $P$, defined in fact on the union $\bigcup U_{i}$.

In $\S 5$ a form of the implicit function theorem was needed which provides for uniformity in the size of the domains of different implicit functions. We now give a precise statement.

THEOREM 10.2. For each $i$ in some index set $I$, let $F_{i}$ and $G_{i}$ be Banach spaces, $E_{i}$ a metric space, $A_{i}$ an open subset of $E_{i} \times F_{i}$ and $f_{i}: A_{i} \rightarrow G_{i}$ a continuous mapping such that $D_{2} f_{i}$ exists and is continuous. Let $\left(x_{0 i}, y_{0 i}\right)$ be a point in $A_{i}$ such that $f\left(x_{0 i}, y_{0 i}\right)=0$ and $D_{2} f_{i}\left(x_{0 i}, y_{0 i}\right)$ is invertible. We make the following uniformity assumptions.

(a) There exists $r>0$ such that $A_{i}$ contains the ball with centre $\left(x_{0 i}, y_{0 i}\right)$ and radius $r$ for all $i$.

(b) There exists $k>0$ such that $\left\|D_{2} f_{i}\left(x_{0 i}, y_{0 i}\right)^{-1}\right\|<k$ for all $i$.

(c) The continuity of $D_{2} f_{i}$ at $\left(x_{0 i}, y_{0 i}\right)$ is uniform with respect to $i$.

(d) The continuity of $f_{i}\left(\cdot, y_{0 i}\right)$ at $x_{0 i}$ is uniform with respect to $i$.

Then there exist $\varepsilon_{1}<r$ and $\varepsilon_{2}<r$ such that, if $U_{i}$ (resp. $V_{i}$ ) is the ball with centre $x_{0 i}$ (resp. $y_{0_{i}}$ ) and radius $\varepsilon_{1}$ (resp. $\varepsilon_{2}$ ), then there is an implicit function $v_{i}: U_{i} \rightarrow V_{i}$ satisfying $v_{i}\left(x_{0 i}\right)=y_{0 i}$ and $f_{i}\left(x, v_{i}(x)\right)=0$ for all $x \in U_{i}$. The implicit function possesses the usual uniqueness property. Moreover the continuity of $v_{i}$ at $x_{0 i}$ is uniform with respect to $i$. Finally if $E_{i}$ belongs to a suitable category which admits a first-order differential operator $L_{i}$, and the continuity of $L_{i} f_{i}$ at $\left(x_{0 i}, y_{0 i}\right)$ is uniform with respect to $i$, then the continuity of $L_{i} v_{i}$ at $x_{0 i}$ is uniform with respect to $i$.

PROOF. For all but the last two sentences the reader is asked to study Dieudonne's proof of the implicit function theorem and observe how the sizes of the neighbourhoods $U$ and $V$ depend on inequalities associated with the norm of $D_{2} f\left(x_{0}, y_{0}\right)^{-1}$, and the continuity of $f\left(\cdot, y_{0}\right)$ and $D_{2} f$.

The penultimate sentence is proved by a trick. Given $\varepsilon<r$ we replace each $A_{i}$ by the ball with centre $\left(x_{0 i}, y_{0 i}\right)$ and radius $\varepsilon$, and use what we have just proved.

To prove the last statement we have that

$$
L_{i} v_{i}(x)=-D_{2} f_{i}\left(x, v_{i}(x)\right)^{-1} L_{i} f_{i}\left(x, v_{i}(x)\right) \text {. }
$$

The result follows since $v_{i}$ is uniformly continuous at $x_{0 i}$, and assumptions (b) and (c) imply that $\left\|D_{2} f_{i}(x, y)^{-1}\right\|$ is uniformly bounded on a neighbourhood of $\left(x_{0 i}, y_{0 i}\right)$ with radius independent of $i$. This concludes the proof.

The following is a formal statement embodying the arguments used in $\S \S 2$ and 5.

THEOREM 10.3. Let $T$ be a metric space, $X$ a Banach space, $A$ an open subset of $T \times X$, and $f: A \rightarrow X$ a continuous mapping such that $D_{2} f$ exists and is continuous. Let $t_{0}$ be a point of $T$ such that $f\left(t_{0}, x\right)=x$ whenever $\left(t_{0}, x\right) \in A$, and suppose that $t_{0}$ has a basis of neighbourhoods $\left\{U_{n}\right\}_{0}^{\infty}$ such that $U_{n+1} \subset U_{n}$ and $U_{n}$ is connected for each $n$. Let $\bar{f}: A \rightarrow T \times X$ be the mapping $(t, x) \mapsto(t, f(t, x))$. Then the following two statements hold, of which the second is a "uniform" version of the first.

(i) There are open subsets $B$ and $C$ of $T \times X$ each containing the set $\{(t, x) \in$ $\left.A: t=t_{0}\right\}$, such that $B$ is contained in $A$ and the restriction $\bar{f} \mid B$ is a homeomorphism of $B$ onto $C$. 
(ii) Let $S$ be a subset of $X$ such that $A$ contains a set of the form $U_{n} \times S_{\varepsilon}\left(S_{\varepsilon}\right.$ stands for the set of points within $\varepsilon$ of $S$ ), and such that the continuity of $D_{2} f$ at $\left(t_{0}, x\right)$ and of $f(\cdot, x)$ at $t_{0}$ is uniform for $x \in S$. Then there exists an open set $D \subseteq A$, a subscript $m$, and $r>0$ such that $\bar{f} \mid D$ is a homeomorphism of $D$ onto $U_{m} \times S_{r}$. Furthermore $D$ contains a set of the form $U_{k} \times S_{\delta}$.

ProOF. We shall only outline the proof. Both parts are based on solving the equation $y=f(t, z)$ for $z$ as a function of $y$ and $t$. We obtain an implicit function in a neighbourhood of each point of the form $\left(t_{0}, x\right)$ in $A$. The implicit functions are glued together using Corollary 10.1 and the ensuing discussion, and the union of their domains is $C$. Then $B$ is the set $\bar{f}^{-1}(C)$.

For the second part we use the uniform implicit function theorem (Theorem $10.2)$ to solve for $z$ in a neighbourhood of each point $\left(t_{0}, x\right)$ such that $x \in S$. We obtain implicit functions defined on domains of uniform size, and gluing these together we obtain $U_{m} \times S_{r}$. Let $h: U_{m} \times S_{r} \rightarrow X$ be the implicit function, that is $x=f(t, h(t, x))$ for all $(t, x) \in U_{m} \times S_{r}$. From the last two sentences of Theorem 10.2 we can conclude that the continuity of $h$ and $D_{2} h$ at points $\left(t_{0}, x\right)$ is uniform for $x \in S$. Then applying to $h$ what we have just proved for $f$, we find that $D$, defined as $f^{-1}\left(U_{m} \times S_{r}\right)$, must contain a set of the form $U_{k} \times S_{\delta}$. This concludes the proof.

\section{REFERENCES}

1. M. Buchner, J. Marsden and S. Schecter, Applications of the blowing-up construction and algebraic geometry to bifuncation problems, Preprint, Univ. of California, Berkeley, 1981.

2. S-N. Chow, J. K. Hale and J. Mallet-Paret, Apptications of generic bifuncation. II, Arch. Rational Mech. Anal. 62 (1976), 209-325.

3. M. G. Crandall and P. Rabinowitz, Bifurcation from simple eigenvalues, J. Funct. Anal. 8 (1971), 321-340.

4. N. H. Kuiper, $C^{1}$-equivalence of functions near isolated critical points, Symposium on InfiniteDimensional Topology, edited by R. D. Anderson, Princeton Univ. Press, Princeton, N.J.; Univ. of Tokyo Press, Tokyo, 1972.

5. T-C. Kuo, Characterisation of $v$-sufficiency of jets, Topology 11 (1972), 115-131.

6. R. J. Magnus, On the local structure of the zero-set of a Banach space vahued mapping, J. Funct. Anal. 22 (1976), 58-72.

7. - The reduction of a vector-valued function near a critical point, Battelle-Geneva Math. Report no. $93,1975$.

8. __ Topological equivalence in bifuncation theory, Functional Differential Equations and Bifurcation, Lecture Notes in Math., vol. 799, Springer, Berlin and New York, pp. 263-276.

9. J. Marsden, Qualitative methods in bifuncation theory Bull. Amer. Math. Soc. 84 (1978), 11251148.

10. M. Shearer, Bifuncation in the neighbourhood of a non-isolated critical point, Israel J. Math. 30 (1978), 363-381.

11. S. Smale, Stable manifolds for differential equations and diffeomorphisms, Ann. Scuola Norm. Sup. Pisa (3) 17 (1963), 97-116.

12. A. Szulkin, Local structure of the zero-sets of differentiable mappings and application to bifurcation theory, Math. Scand. 45 (1979), 232-242.

13. C. A. Stuart, Bifuncation for variational problems when the linearisation has no eigenvalues, J. Funct. Anal. 38 (1980), $169-187$.

14. J. Dieudonné, Foundations of modern analysis, Academic Press, New York and London, 1960.

DEPARTMENT OF MATHEMATICS, SCIENCE INSTITUTE, UNIVERSity OF ICELAND, DUNHAGA 3, IS-107 REYKJAVIK, ICELAND 